\title{
Les faits de corruption à l'échelle internationale : le rôle des pouvoirs publics canadiens et des outils de soft law
}

\author{
Benoit Lapointe $^{a}$, Jeanne Simard $^{b}$, Marc-André Morency $^{c}$, Salmata Ouedraogo $^{d}$
}

RÉSUMÉ. Toute action efficace visant la corruption internationale requiert l'établissement en infraction pénale des comportements visés et la mise en œuvre de mesures répressives destinées à rendre ces pratiques moins attractives. C'est le modèle qu'a instauré, en 1998, le Canada, en adoptant la Loi sur la corruption des agents publics étrangers et en modifiant, en 2004, le Code criminel afin que la responsabilité des organisations soit désormais établie en fonction des actes et des intentions de leurs « cadres supérieurs ». De façon parallèle, différents organismes multilatéraux (l'OCDE, l'ONU, le G20) ont élaboré au fil des ans des normes anticorruption afin de promouvoir de meilleures pratiques en matière de responsabilité sociale au sein des entreprises multinationales. Mais au-delà de l'adoption d'une régulation étatique, multilatérale ou internationale, le succès de la lutte à la corruption parait également résider dans l'adoption, au sein même des organismes et des entreprises actives à l'échelle internationale, d'une politique de conformité et d'un système de gestion interne dont l'objet est de prévenir, détecter et lutter contre les faits de corruption. Cela requiert plus largement de modifier la conscience des acteurs sociaux en favorisant l'instauration d'une culture organisationnelle plus éthique, du haut en bas de l'entreprise, mais aussi plus transparente autant du côté des opérateurs économiques que des pouvoirs publics.

$\boldsymbol{A B S T R A C T}$. Effective action against international corruption requires the criminalization of the targeted behaviors and the enforcement of repressive measures to make such practices less attractive. This is the model adopted by Canada in 1998 through the Corruption of Foreign Public Officials Act and through amending the Criminal Code in 2004, establishing accountability for the acts of organizations and for the intentions of their "senior officers". In parallel, different multilateral agencies (the OECD, the UN, the G20) have developed anti-corruption standards over the years to promote better social responsibility practices within multinational corporations. Beyond the adoption of state, multilateral or international regulation, the success of the fight against corruption also seems to lie in the adoption, within organizations and companies active at the international level, of a compliance policy and an internal management system whose purpose is to prevent, detect and fight against corruption. This requires more broadly to alter the consciousness of social actors via the establishment of a more ethical organizational culture, from top to bottom in a company, but also a more transparent culture on the part of economic operators and public authorities.

L'entreprise contemporaine et ses gestionnaires sont aujourd'hui confrontés à de nombreux risques pour leur réussite en raison de la complexité des situations auxquelles ils doivent faire face. Des objectifs souvent divergents sollicitent leur attention : rendement, sécurité, pouvoir, adaptation au contexte, etc. Parmi ces risques, les faits de corruption posés par les dirigeants, cadres ou employés, ainsi que par différents partenaires contractuels, au niveau national et à l'international, demeurent ardus à gérer en raison du secret qui les entoure et des effets très néfastes qu'ils peuvent avoir sur la réputation ou la marque de l'entreprise. La confiance des partenaires peut s'effriter et la mise à l'écart de certains marchés publics pourrait affecter la valeur et la viabilité à long terme de l'entreprise.

\footnotetext{
a Chargé de cours, Université du Québec à Chicoutimi

b Professeure, LL D., avocate, F. Adm.A., Université du Québec à Chicoutimi

c Professeur retraité, sociologue, Université du Québec à Chicoutimi

d Professeure, Ph. D., Université du Québec à Chicoutimi
} 
Toute action efficace visant la corruption internationale requiert au départ l'établissement en infraction pénale des comportements visés et la mise en œuvre de mesures répressives destinées à rendre ces pratiques moins attractives. C'est le modèle qu'a instauré aux États-Unis le président Carter en décembre 1977 en promulguant le Foreign Corrupt Practices Act. Deux décennies plus tard, le Canada adoptait, en 1998, la Loi sur la corruption des agents publics étrangers (LCAPE). Il a aussi modifié en 2004 le Code criminel afin que la responsabilité des organisations soit désormais établie en fonction des actes et des intentions de leurs « cadres supérieurs ». Le 1er juillet 2011, le Royaume-Uni a pour sa part adopté le Bribery Act 2010, qui durcit le droit britannique envers les pratiques de corruption étrangères. De façon parallèle, différents organismes multilatéraux ('OCDE, l'ONU, le G20) ont élaboré au fil des ans des normes anticorruption afin de promouvoir de meilleures pratiques en matière de responsabilité sociale au sein des entreprises multinationales.

Mais au-delà de l'adoption d'une régulation étatique, multilatérale ou internationale, le succès de la lutte à la corruption parait également résider dans l'adoption, au sein même des organismes et des entreprises actives à l'échelle internationale, d'une politique de conformité et d'un système de gestion interne dont l'objet est de prévenir, détecter et lutter contre les faits de corruption. Cela requiert plus largement de modifier la conscience des acteurs sociaux en favorisant l'instauration d'une culture organisationnelle plus éthique, du haut en bas de l'entreprise, mais aussi plus transparente autant du côté des opérateurs économiques que des pouvoirs publics.

Le présent article a pour objectif de dégager un aperçu général des phénomènes de corruption au niveau international. Plus spécifiquement, on tentera, dans un premier temps, d'en définir l'étendue dans son contexte social global (1). Dans un deuxième temps, nous ferons état des réactions sur le plan normatif au niveau international ainsi qu'au Canada et nous en soulignerons les limites (2). Dans un troisième temps, nous examinerons plus spécifiquement l'importance des instruments de soft law en tant qu'instruments de prévention et de détection des faits de corruption, notamment les codes de conduite adoptés par les entreprises, les engagements des dirigeants à cet effet, les programmes de communication et de formation, les pactes d'intégrité, les politiques de gestion du risque et les mécanismes de dénonciation (3). Nous verrons que sur le plan concret, la corruption, avec son caractère multiforme, demeure toujours très difficile à évaluer avec précision et encore plus à contrôler malgré les efforts déployés par les États ou les organisations multilatérales. C'est un chantier dynamique et en constante évolution, qui requiert une attention soutenue des entreprises, des gestionnaires et des agences de répression.

\section{La corruption dans le monde : définition, statistiques et aspects sociaux économiques}

Les faits de corruption sont vieux comme le monde. Déjà dans l'Antiquité, Platon et Aristote utilisaient ce terme "pour qualifier la santé morale de la société» (Perrin, 2010, p. 87; Ménissier, 2007). Pour Montesquieu « la corruption de chaque gouvernement commence prefque toujours par celle des principes ». Par exemple, «le principe de la démocratie fe corrompt, non feulement, lorfqu'on perd l'efprit d'égalité, mais encore quand on prend l'efprit d'égalité extrême, \& que chacun veut être égal à ceux qu'il choifit pour lui commander» (Montesquieu, 1772, p. 225). À notre époque, la corruption est un phénomène exacerbé par les caractères de la mondialisation, et la recherche sans limites de la maximisation du profit (Kartz, 2011). De nombreux cas de corruption sont rapportés dans les médias presque quotidiennement dans divers secteurs de l'économie, dans les industries extractives, la construction, le transport, la finance, le sport ou encore les communications. Mais comment définir la corruption? Qu'implique-t-elle?

\subsection{Définition de la corruption}

Le terme vient du latin "corrumpere », qui signifie «briser complètement, détériorer physiquement ou moralement ». Plus simplement, un acte de corruption implique un échange occulte, un abus de pouvoir à des fins privées (Rodriguez, Siegel, Hillman et Eden, 2006). La corruption qualifie l'acte d'un «agent» qui, en agissant dans son intérêt personnel, trahit (brise) la confiance mise en lui par la personne publique ou privée (le principal) dont les intérêts devraient normalement être protégés par l'agent en raison du mandat qu'il a reçu d'agir au mieux de ses intérêts. L'agent utilise le pouvoir 
dont il dispose afin de donner un traitement préférentiel déraisonnable à un corrupteur en contrepartie d'un avantage personnel qui peut être ou non financier.

Parmi les pratiques les plus courantes de corruption, on trouve « les paiements en espèces ou autres pour obtenir un contrat gouvernemental ou commercial, les contributions politiques ou charitables inappropriées, les pots-de-vin, les dons excessifs et les paiements illicites pour obtenir un permis ou d'autres mesures réglementaires » [notre traduction] (Kartz, 2011, p. 502).

La corruption est un phénomène complexe, et pour en saisir les multiples dimensions, il importe d'en distinguer les référentiels.

\section{Corruption publique et corruption privée}

La référence aux fonctions étatiques qu'implique la corruption dite " publique » la singularise. Elle concerne deux aspects en particulier : d'abord la fonction de la personne susceptible d'être corrompue, puis le comportement qui est attendu d'elle par l'autorité de tutelle. Par conséquent, pour qu'il y ait corruption publique, la situation doit impliquer une personne qui occupe une fonction publique quelconque, relevant de l'ordre législatif (députés), exécutif (ministres, maires, fonctionnaires) ou judiciaire. Il faut en outre que l'acte abusif entre dans cette sphère publique, c'est-à-dire qu'il est posé dans l'exercice ou à l'occasion de l'exercice de cette fonction. Ceci signifie que l'acte accompli par la personne publique doit avoir un lien quelconque, direct ou indirect, avec sa fonction.

À l'opposé, la corruption dite «privée » a trait aux agissements de deux agents (cadres, employés) dans le cadre d'une activité privée, de caractère généralement commercial ou industriel. Elle porte habituellement sur l'attribution de contrats, à savoir que, pour se les voir attribuer, l'agent de l'entreprise désireuse de les obtenir, va fournir à un agent de l'autre entreprise un avantage quelconque de caractère personnel n'ayant rien à voir avec le prix ou les autres conditions contractuelles, et ceci dans le but d'influer sur la décision définitive. La différence essentielle par rapport à la corruption publique est que les entreprises impliquées sont toutes à capitaux privés, et qu'il n'y a aucun intérêt public lié à l'attribution des contrats faisant l'objet d'actes de corruption.

\section{Corruption internationale et corruption nationale}

La corruption sera dite internationale lorsqu'elle comporte un élément d'extranéité : par exemple, lorsque l'acte de corruption a lieu entre deux agents appartenant à deux pays différents, ou encore lorsque l'un des actes constitutifs d'un comportement de corruption se situe dans un autre État. Ce serait le cas, par exemple, si le pacte de corruption a été conclu dans un hôtel situé dans une ville d'un État étranger, ou encore si l'argent devant être remis au corrupteur passif a transité par un compte bancaire situé dans une banque localisée dans un État étranger. En revanche, la corruption sera dite nationale si elle ne comporte aucun élément d'extranéité, dans le sens où tous les éléments constitutifs d'actes de corruption se limitent ou se déroulent sur le territoire d'un seul État.

\section{Corruption active et corruption passive}

La pratique permet de faire la distinction entre la corruption active et la corruption passive. La première se définit comme l'offre, la promesse ou l'octroi de tout avantage à une personne (agent public ou privé) pour qu'elle agisse en contravention des obligations de sa fonction afin de satisfaire l'intérêt personnel de celui qui compte la faire agir autrement que dans l'intérêt normalement prédominant de son principal (ou commettant). La corruption passive a trait au comportement de l'agent qui reçoit, ou encore sollicite le versement d'un avantage indu de la part d'individus ou d'entreprises, en l'occurrence étrangers.

Corruption comme simple transaction et corruption en réseau

Il faut également, pour concevoir adéquatement les phénomènes de corruption, réaliser qu'ils ne sont pas toujours simplement de simples transactions entre un corrupteur (offre) et un agent public corrompu (demande), car, aujourd'hui, il arrive que ce dernier se situe du côté de l'offre. Le caractère de ces actes est en effet souvent beaucoup plus complexe, s'inscrivant dans des réseaux qui échappent aux acteurs individuels. Plusieurs intermédiaires peuvent intervenir dans la transmission ou la réception du pot-de-vin.

$[\ldots]$ il n'y a plus alors de relation proprement dite entre «un» corrupteur et «un» corrompu, mais un entrelac complexe de relations entre des personnes redevables les unes 
envers les autres; en d'autres mots, un échange informel d'aides éparses au profit de gens dont chaque membre du réseau ne sait rien... sinon qu'ils font partie du réseau! (Perrot, 2016).

Enfin, la corruption se diversifie. Par exemple, il peut arriver qu'aucun montant ne soit versé, la rétribution de l'agent corrompu se matérialisant, par exemple, dans un cas de corruption privée, par le fait que l'entreprise corruptrice obtiendra non pas le contrat pour lequel elle corrompt, mais un autre contrat qui sera attribué dans l'avenir.

\section{Corruption systémique}

La corruption, au-delà des actes posés par des individus, trouve la possibilité d'exister au sein des institutions politiques et économiques d'un pays. Elle est alors engendrée au sein de vastes systèmes de rapports sociaux, un tout global affectant généralement les activités humaines, et les institutions démocratiques. L'universalité d'un tel phénomène conduit le juge Garapon à montrer le caractère surdéterminant de la corruption, son aspect systémique :

La corruption, c'est un crime du deuxième degré : non pas un crime contre les personnes ou les biens, mais un crime contre le droit; et s'il fait des victimes, ce sont des victimes indirectes qui sont beaucoup plus nombreuses que n'en fait n'importe quel autre crime, car il s'agit de la collectivité politique tout entière (Garapon, 2014, p. 24).

D'autres auteurs viennent confirmer cette représentation :

Dans le cas systémique, la corruption imprègne toute la société et, dans le processus, devient routinière et acceptée comme un moyen de mener des transactions quotidiennes. Cette corruption « enracinée » ou « systémique » affecte les institutions et influence le comportement individuel à tous les niveaux du système politique et socio-économique (Johnston, 1996). La corruption enracinée caractérise les sociétés suivantes : faible concurrence politique, croissance économique faible et inégale, société civile faible et absence de mécanismes institutionnels pour lutter contre la corruption (Robinson, 2004) [notre traduction] (Ampratwum, 2008, p. 77).
Par ailleurs, l'économiste François Morin (2015) décrit la dominance d'une quinzaine d'oligopoles financiers ou bancaires constituant l'équivalent d'un Parlement mondial; ces oligopoles sont considérés «too big to fail», et les bulles spéculatives dont elles sont responsables, grâce aux dérégulations engagées depuis les années 1980, ont forcé les États à absorber leur sauvetage à plusieurs reprises : la dette des États à leur endroit représente le coût de cette corruption systémique que ces acteurs publics et privés entretiennent de façon incestueuse entre eux, le régulé devenant le régulateur... ou le dérégulateur!

Plus encore, selon une équipe de la Swiss Federal Institute of Technology de Zurich, 147 firmes contrôlent $40 \%$ du commerce mondial (Vitali, Glattfelder et Battiston, 2011). L'étude dévoile la structure en réseau de quelque 700 firmes majeures, leur capacité de contrôler la production et les échanges à l'échelle mondiale. Elle met aussi en évidence un réseau de 50 sociétés transnationales dont le contrôle est susceptible d'affecter la concurrence sur le marché mondial ainsi que sa stabilité financière. Seuls de petits échantillons nationaux avaient auparavant été étudiés et il n'y avait aucune méthodologie appropriée pour évaluer un tel contrôle à l'échelle mondiale. Cette recherche a révélé que les sociétés transnationales forment une structure en forme de nœud papillon géant et qu'une grande partie du contrôle des flux prend sa source dans un petit noyau très soudé d'une quinzaine d'institutions financières et bancaires, ce que confirme Morin (2015). Ce noyau peut être considéré comme une « superentité » économique, ce qui soulève de nouvelles questions importantes à la fois pour les chercheurs et les décideurs politiques. Ces recherches illustrent le caractère mythique des théories économiques centrées sur un concept de concurrence anhistorique et de perfection des marchés (Boyer, 2015). Cette évolution conduit à interroger la nature même du fait corporatif et ses dérives dans le contexte de la mondialisation.

\subsection{La corruption internationale en chiffres et ses effets}

Pour de nombreux économistes, la corruption plonge ses racines dans la délégation de pouvoir à l'origine du fait corporatif lui-même. C'est l'usage discrétionnaire du pouvoir dont jouissent certains 
agents économiques qui rend possible la corruption. Bien qu'il soit difficile de calculer les coûts exacts de la corruption, du fait du secret qui entoure les différentes opérations, certains de ses effets sont visibles, des effets économiques et politiques aussi bien sur le plan macroéconomique que microéconomique. En effet, la corruption diminue l'efficacité économique. Depuis plusieurs années, des chercheurs se consacrent à l'analyse de ses effets. Paolo Mauro (1997) a montré que la corruption réduit le montant global des investissements des pays. Une étude se concentrant sur 67 pays pour la période de 1960 à 1985 a montré que la réduction des activités de corruption influe sur les flux d'investissements et se traduit par une hausse du taux d'investissement. Il montre que, si l'Égypte améliorait son efficacité administrative et réduisait son niveau de corruption, les investissements augmenteraient de $3 \%$ et le taux de croissance de $0,5 \%$. Notons que selon l'indice de perception de la corruption de Transpareny International, l'Égypte, avec un score de 32 (un score de 100 signifie 0 corruption), est classée $117^{\mathrm{e}}$ sur 180 pays. Wei (2000) abonde dans le même sens et montre que la corruption a pour effet de rendre inefficaces les dépenses publiques puisqu'elles sont détournées vers des projets non socialement rentables par le canal des paiements facilitateurs ou pots-de-vin. En créant l'incertitude pour les investisseurs et les organisations, la corruption détériore l'environnement des échanges commerciaux.

Sur le plan politique, la corruption encourage l'émergence d'institutions politiques extractives, c'est-à-dire des institutions économiques qui retirent les ressources de l'économie en ne protégeant pas les droits de propriété, en n'appliquant pas les contrats privés, en interférant avec les marchés. Ce sont des institutions construites de manière à extraire les ressources du reste de la société. Transparency International, une organisation non gouvernementale consacrée à la lutte contre la corruption, dans son rapport sur la perception de la corruption, montre l'existence de liens entre les niveaux de corruption, la défense des droits et libertés et la mobilisation de la société civile (Transparency International, 2017). La corruption influe alors sur la qualité de la démocratie dans les différents pays.

Aujourd'hui, les investissements directs étrangers (IDE) sont un élément moteur de la multinationalisation des entreprises et de la mondialisation.
Grâce aux politiques macroéconomiques favorables, les IDE ont connu une croissance considérable au cours des dernières décennies, mais cet essor a également été accompagné d'une augmentation significative de la corruption (Sanyal, 2005). Ces 25 dernières années, les IDE ont connu une croissance phénoménale, mais une baisse est survenue en 2016. « Les flux mondiaux d'IDE devraient atteindre près de 1800 milliards de dollars en 2017 et 1850 milliards de dollars en 2018 » (Conférence des Nations Unies sur le commerce et le développement, 2017). Dans un rapport publié en 2014, l'Organisation de développement et de coopération économique (OCDE) signale l'ampleur de la corruption internationale. Le rapport analyse plus de 400 cas de corruption survenus dans le monde, impliquant aussi bien des personnes morales que physiques. Une des principales conclusions est que deux tiers des affaires de corruption sont survenus dans quatre secteurs seulement: les industries extractives (dans 19\% des cas), la construction $(15 \%)$, le transport et l'entreposage $(5 \%)$ et le secteur de l'information et de la communication $(10 \%)$ (OCDE, 2014).

Le fouillis de comportements corrompus fait qu'il est impossible de les connaitre avec certitude, tout comme le coût réel de la corruption, en raison de sa clandestinité et de sa structure en réseau. Différentes approches ont été développées depuis ces dernières années pour contourner la difficulté. On trouve des mesures basées sur l'étendue de la corruption, sur le coût de la corruption et les tendances en matière de corruption. Dans tous les cas, des problèmes conceptuels et méthodologiques rendent l'interprétation directe très difficile (Barkemeyer, Preuss et Lee, 2015; Heywood et Rose, 2014).

Faute de pouvoir documenter tous les faits de corruption, on peut évaluer les perceptions, de manière à en apprécier l'incidence. C'est le rôle d'un indice de perception (IPC) qui se construit à partir de plusieurs sondages d'opinion d'experts. Ces derniers, à partir de leur perception du niveau de corruption dans le secteur public d'une centaine de pays, les classent sur une échelle de 0 à 100; 0 indiquant un degré élevé de perception de la corruption et 100 indiquant un pays sans corruption. À titre d'exemple, selon l'indice de perception de la corruption (IPC) en 2017 de Transparency International, dans deux tiers des 180 pays/territoires l'ampleur 
du fardeau de la corruption est importante, ces pays enregistrant une note inférieure à 50 . Le rapport de 2017 estime que dans les pays pauvres, l'ampleur de la corruption est importante et s'y trouve couplée avec l'instabilité politique ou sociale. L'IPC permet

\begin{tabular}{|c|c|c|c|}
\hline \multicolumn{2}{|c|}{ Pays perçus comme les moins corrompus } & \multicolumn{2}{|c|}{ Pays perçus comme les plus corrompus } \\
\hline Rang & Pays & Rang & Pays \\
\hline 1 & Nouvelle-Zélande & 168 & Vénézuela \\
\hline 2 & Danemark & 171 & Corée du Nord \\
\hline 3 & Finlande & 171 & Libye \\
\hline 4 & Norvège & 171 & Guinée-Bissau \\
\hline 5 & Suisse & 175 & Yémen \\
\hline 6 & Singapour & 175 & Soudan \\
\hline 7 & Suède & 177 & Afghanistan \\
\hline 8 & Canada & 178 & Syrie \\
\hline 8 & Luxembourg & 179 & Sud Soudan \\
\hline 8 & Pays-Bas & 180 & Somalie \\
\hline
\end{tabular}

Tableau 1 - Classement des pays perçus comme les plus corrompus et les moins corrompus à travers le monde Source : Construction des auteurs à partir des données de Transparency International (2017)

Ce niveau élevé de perception de la corruption met à mal les abstractions économiques des théories sur le fonctionnement des marchés. Les acteurs sociaux sont-ils égaux? Leur rationalité est-elle parfaite? Ces indices de perception de la corruption ne résolvent pas la question de la fiabilité de la perception (Dion, 2013). L'information est-elle accessible à tous les acteurs sociaux? En ce cas, comment le comportement économique pourrait-il être aussi rationnel qu'on l'entend dans la théorie économique?

\subsection{Le coût de la corruption dans le monde}

Des recherches de mieux en mieux documentées estiment que le coût de la corruption s'élève à des centaines de milliards de dollars chaque année, soit une fraction importante du produit économique global, ce qui en fait un problème global des plus pressants à régler : de dévoiler l'ampleur de la corruption et de sonner l'alarme sur la corruption endémique dans certains pays. La figure suivante donne un aperçu des faits de corruption dans le monde (Transparency International, 2017) 1 
inégalités, les pauvres et les femmes en sont les principales victimes, tandis que l'instabilité touche les plus hauts sommets des États [...] (Sancerre, 2016, FMI, 2016).

En avril 2018, le FMI adoptait un cadre réglementaire, applicable à partir du 1 er juillet 2018, qui permettra d'évaluer "la corruption systématique» dans les 189 pays membres. Le cadre souligne que la corruption nuit à la croissance économique, aux investissements et aux recettes fiscales, le tout sur fond de faiblesses dans la gouvernance des organisations gouvernementales et privées :

«L'accent est mis sur la gouvernance de façon globale, et pas seulement sur la corruption ». Car «les faiblesses de gouvernance [...] ouvrent généralement des portes à la corruption » (Agence France-Presse, 2018).

Selon la Commission économique des Nations Unies pour l'Afrique (CEA), l'Afrique perd environ 148 milliards de dollars chaque année en lien avec la corruption. Ce chiffre représente environ $25 \%$ du PIB moyen d'un continent affligé de tous les maux (pauvreté, inégalités, migrations, etc.) (Atcha, 2018).

Une estimation des pertes pour le monde et l'Europe est considérée par Bonell et Meyer comme particulièrement lourde :

Corruption is said to increase the total cost of doing business globally by up to $10 \%$ and the cost of procurement contracts in developing countries by up to $25 \%$. This means that for the EU alone approximately EUR120 billions, or $1 \%$ of its GDP, is lost to corruption every year (Bonell, Meyer, 2015, p. 2).

En synthèse, on peut estimer que le taux de croissance annuel de l'économie mondiale est pratiquement mangé par la corruption, contribuant de façon significative à exacerber les inégalités sociales sur la planète et à gangrener les institutions économiques et politiques.

\subsection{Les aspects sociaux et culturels de la corruption internationale}

Une interprétation laxiste se réclamant du relativisme culturel voudrait que les faits de corruption soient légitimes lorsqu'ils s'inscrivent dans le cadre des pratiques d'affaires habituelles d'un secteur d'activité ou font partie de la culture d'un État (Akbar et Vujić, 2014; Husted, 1999). On prétend en cela que la corruption doit être envisagée relativement à la culture nationale au sein de laquelle elle s'exerce, certaines pratiques étant acceptables pour un État et non pour d'autres. Bref, la pratique et l'usage se feraient doctrine!

Pour illustrer cette dérive normative, la littérature donne souvent comme exemple la pratique du guanxi, en Chine. Le guanxi implique un échange de faveurs entre partenaires d'affaire visant à créer progressivement la confiance mutuelle nécessaire à l'établissement de liens contractuels; cette pratique est bien souvent associée à de la corruption dans certains États développés où la culture du contrat relationnel s'inscrit dans une longue tradition. Le guanxi serait ainsi nécessaire pour pénétrer le marché chinois et y maintenir sa présence.

Les entreprises sont de fait placées dans des situations délicates quand ces cadeaux ou échanges de faveurs sont perçus comme une composante de l'étiquette des affaires d'un secteur d'activité spécifique, ou encore lorsqu'ils sont inséparables de la culture ou de l'histoire de l'État dans lequel ces pratiques prennent place. C'est le cas, par exemple, dans les États arabes où règnent des usages pouvant s'apparenter, d'une certaine manière, à ceux ayant cours en Chine, et qu'on désigne sous l'appellation de wasta. La remarque s'applique de façon plus générale à l'égard de tous les États que l'on classe, en management international (ou interculturel - cross-cultural management), dans la catégorie des bigh-context cultures, c'est-à-dire les cultures dans le cadre desquelles, parmi d'autres caractéristiques liées au contexte, l'établissement de liens interpersonnels est un préalable indispensable à la concrétisation de tout lien d'affaires (Harrison, 2014). En outre, les cultures nationales caractérisées par un haut degré d'acceptation de la hiérarchie dans les rapports sociaux (power distance) seraient plus enclines à accepter les pratiques de corruption que celles qui sont davantage marquées par une recherche d'égalité dans les relations sociales.

C'est dans ce contexte qu'il convient d'apprécier les efforts destinés à faire évoluer les pratiques sous l'égide d'un droit international ou national soucieux de réduire la masse des dérives que ces traditions, ou ce relativisme, continuent d'alimenter. 


\section{L'évolution du droit international et du droit canadien en matière de corruption internationale}

À l'encontre de la doctrine et surtout des pratiques de laisser-faire relevant du paradigme relativiste, depuis 30 ans, de nombreuses initiatives ont été prises, autant au niveau international, que national ou régional, pour lutter contre la corruption. Cette coopération entre les États nationaux, les organismes multilatéraux et les entreprises a permis d'élaborer de nombreux instruments normatifs. Cette mobilisation était nécessaire, car la corruption ne connait pas de frontières, n'admet aucune distinction économique et touche toutes les formes de gouvernement (Quinones, 2000). L'intervention des États relativement à la corruption internationale est, pour la plupart d'entre eux, toute récente, mais elle permet d'apprécier la portée de quelques-unes de ces normes et de leur effet catalyseur dans le renforcement des outils internes anticorruption au sein des entreprises opérant dans le contexte mondial (Tomasic, 2018).

\subsection{Le droit international}

L'analyse de la loi américaine, le Foreign Cormpt Practices Act (FCPA), représente le point de départ obligé de toute étude sérieuse des efforts destinés aujourd'hui au niveau international à combattre la corruption d'agents publics étrangers ${ }^{2}$. Avec l'adoption de cette loi en 1977, les États-Unis ont établi la première prohibition législative visant la corruption d'agents publics étrangers. Auparavant, les lois interdisant la corruption publique dans les autres États visaient uniquement les agents nationaux, non ceux d'États étrangers. Les entreprises, notamment américaines, ayant des activités à l'étranger suivaient, à défaut d'une loi nationale portant sur cette question, les coutumes ayant cours dans les États dont relevaient les agents publics en ce qui concerne la légalité du versement de pots-de-vin, pratique souvent acceptée ou tolérée.

Depuis l'adoption et l'entrée en vigueur du FCPA et jusqu’à la fin des années 1990, les États-Unis furent le seul État dans le monde où il était illégal de verser des pots-de-vin à des agents publics étrangers. Cette situation a engendré des tensions et des situations conflictuelles avec certains autres États membres de l'Organisation de coopération et de développement économiques (OCDE), notamment la
France, l'Allemagne et le Japon, ces États permettant, sur le plan fiscal, la déduction des pots-de-vin des revenus des entreprises qui les versaient (OCDE, 2001).

For a variety of reasons, many states have been slow to follow-on from the American legislative and regulatory lead in dealing with foreign bribery. But not all countries have had the international leverage of the USA in imposing anti-bribery measures. This is not surprising given the hegemonic political and economic power of the United States which has enhanced the effectiveness of efforts by the United States to extra-territorially apply anti-bribery laws (Tomasic, 2018, p. 19).

Afin de créer des conditions de concurrence équitables dans l'environnement des affaires internationales, la Convention sur la lutte contre la corruption d'agents publics étrangers dans les transactions commerciales internationales a été adoptée à l'OCDE en novembre 1997. Cette convention a été promue principalement par les États-Unis «qui souhaitaient que leurs partenaires commerciaux soient assujettis à une législation sur le modèle du Foreign Corrupt Practices Act » (Quinones, 2003, p. 564-565). Les 35 États membres de l'OCDE (dont le Canada), ainsi que 8 États non membres (l'Afrique du Sud, l'Argentine, le Brésil, la Bulgarie, la Colombie, le Costa Rica, la Lituanie et la Russie) ont ratifié cette convention. Il s'agit du premier et du seul instrument international de lutte contre la corruption ciblant «l'offre» de pots-de-vin à des agents publics étrangers. L'objectif essentiel de la convention consiste à ériger en infraction pénale, dans chaque État national ayant ratifié cette convention, la corruption à la source, en pénalisant le «corrupteur actif», soit celui qui verse un pot-de-vin à un agent public étranger (D’Hollander, 1999; Cavalerie, 1997). Elle ne vise donc pas la corruption passive internationale. Pour cela, il faudrait que les États incriminent le comportement de fonctionnaires étrangers, ce qu'ils n'ont pas la compétence de faire, car ils n'ont aucun lien de rattachement avec eux. Seuls les États dont ces fonctionnaires relèvent peuvent les sanctionner en raison du lien tiré de la nationalité qui leur donne la compétence dite personnelle. La compétence des États signataires de la convention de l'OCDE ne peut, en l'occurrence, que s'étendre aux actes commis à l'étranger par les entreprises qui détiennent leur nationalité ou qui, par ailleurs, relèvent de leur droit. 
Il faut par ailleurs souligner, à l'échelle transnationale, la signature, le 9 décembre 2003, à Mérida (Mexique), par 114 États, de la Convention des Nations Unies contre la cormption (CNUCC) (Office des Nations Unies contre la drogue et le crime, 2004) ${ }^{3}$. Il s'agit, au niveau mondial, de la convention la plus complète sur le sujet, autant sur le plan du droit substantif que procédural ${ }^{4}$. Les objectifs de la CNUCC sont mentionnés clairement à l'article 1 :

a) De promouvoir et renforcer les mesures visant à prévenir et combattre la corruption de manière plus efficace; b) De promouvoir, faciliter et appuyer la coopération internationale et l'assistance technique aux fins de la prévention de la corruption et de la lutte contre celleci, y compris le recouvrement d'avoirs; c) De promouvoir l'intégrité, la responsabilité et la bonne gestion des affaires publiques et des biens publics.

La faiblesse de la CNUCC tient cependant au fait qu'elle combine à la fois des dispositions formulées dans des termes contraignants et des dispositions de « droit mou ».

La Convention contraint les pays à créer des infractions criminelles ou d'autres types d'infractions pour toute une gamme d'actes de corruption, si ces derniers ne sont pas déjà considérés comme des crimes par le droit interne. Dans certains cas, les États ont l'obligation légale de créer des infractions; dans d'autres cas, afin de tenir compte des différences d'ordre juridique d'un pays à l'autre, ils doivent envisager de le faire (Becklumb, 2007, p. 5).

C'est pourquoi il ne convient pas de comparer la CNUCC à la convention de l'OCDE de 1997, en dépit du champ d'application matériel et spatial (ou géographique) plus limité de cette dernière.

Par ailleurs, pour les 20 plus grandes puissances économiques, membres du G20, la prévention et la réduction de la corruption demeurent une priorité d'action sur le plan international. On a adopté, en juin 2010, lors du sommet à Toronto, un plan d'action anticorruption qui a été réaffirmé en 2014 et en 2017 lors du sommet des chefs d'État et de gouvernement tenu à Hangzhou (République populaire de Chine). Ce plan rappelle clairement que la corruption «sape la bonne gouvernance, érode la confiance que les gens placent dans les institutions publiques, corrode la prise de décision, entrave le développement économique et facilite le crime organisé » (G20, 2016). On invite aussi les pays qui ne l'ont pas encore fait à ratifier et à appliquer la Convention des Nations Unies contre la corruption (CNUCC). Un Groupe de travail anticorruption du G20 (GTCA ou ACWG), mis sur pied dès 2010, a engagé une vaste réflexion sur les progrès réalisés dans la lutte contre la corruption à travers le monde et identifié les actions prioritaires pour les prochaines années (G20 Anti-Corruption Working Group, 2017). Ce groupe travaille en coopération avec plusieurs organisations internationales dont l'ONUDC, le Fonds monétaire international (FMI), l'OCDE et la Banque mondiale.

\subsection{Le droit canadien}

L'activité économique au Canada est vulnérable à la corruption, en raison de sa présence importante dans des secteurs à haut risque, comme l'industrie de la construction et l'extraction des matières premières, notamment en pays étrangers. Pourtant, ce n'est qu'en 1998 que le législateur canadien a adopté la Loi sur la corruption des agents publics étrangers (LCAPE) ${ }^{5}$ « en vue d'incorporer dans le droit canadien ses obligations au titre de la Convention [de l'OCDE] » (Gouvernement du Canada, 2017). Auparavant, le Code criminel ne contenait que des dispositions visant la corruption des agents publics nationaux (art. 119 à 125) et les commissions secrètes (art. 426).

\subsubsection{Loi sur la corruption des agents publics étrangers (LCAPE)}

La LCAPE interdit aux personnes physiques et aux personnes morales canadiennes d'offrir, de promettre, de payer ou de donner de l'argent ou des biens de valeur à des agents publics étrangers afin d'obtenir ou de conserver un marché, un contrat ou un avantage injustifié. Quiconque commet ce type d'infraction est coupable d'un acte criminel passible d'un emprisonnement maximal de 14 ans (art. 3). L'interdiction vise les avantages offerts ou donnés aux législateurs, aux juges, à toute personne investie d'une autorité administrative au sein d'un État étranger, aux employés des conseils, des commissions et des sociétés d'État exerçant des fonctions au nom d'un État étranger. La notion d'« État étranger » s'étend à leurs subdivisions politiques, comme les villes ou les provinces, ainsi que leurs organismes. 
La LCAPE interdit de façon évidente le versement de pots-de-vin, mais aussi l'octroi de prêts, récompenses ou avantages de quelque nature que ce soit, par exemple, des voyages, que ce soit afin d'obtenir ou renouveler un contrat commercial international, éviter de l'impôt, obtenir un permis sans respecter les exigences ou payer des droits sur les ressources naturelles (Katz, 2011). Toutefois, sur le plan pratique, nous sommes invités à la nuance, car « cette infraction inclut plusieurs éléments techniques qui en élargissent ou en limitent la portée » (Eljarrat et Bodrug, 2018, p. 1). D'abord, une infraction de corruption peut être commise même si aucun avantage n'est donné dans les faits. Le simple fait d'offrir un tel avantage est prohibé. De même, donner ou offrir de donner par l'intermédiaire d'une tierce personne un avantage interdit constitue également une infraction. L'infraction de corruption peut viser des avantages non pécuniaires, comme la gratuité du logement ou des études ou encore l'allègement des frais liés à ceux-ci.

En 2013, la LCAPE a subi une modification importante à la suite des recommandations des membres du Groupe de travail de l'OCDE sur la corruption lequel jugeait que la loi canadienne n'était pas suffisamment sévère et surtout trop peu appliquée par les autorités canadiennes (OCDE, 2011). Avec cet amendement, on prévoit désormais des peines maximales d'emprisonnement plus sévères (de 5 à 14 ans) et des amendes illimitées pour l'infraction de corruption d'agents publics étrangers. La loi reconnaît aussi une nouvelle infraction dans la situation où une société s'engage dans des pratiques comptables conçues pour dissimuler une activité corrompue (a. 4[1]).

It expands the jurisdiction of the RCMP to investigate not only potential violations of the primary offence, but also the accounting practices, bookkeeping, standards and records of organizations in Canada and abroad (Keith, 2017, p. 57).

La LCAPE élimine par ailleurs l'exception relative à certains types de paiements de facilitation. Ces actes, que certains qualifient de "petite corruption ou de corruption mineure ", se présentent comme des paiements versés afin de hâter ou de garantir l'exécution par un agent public étranger d'un acte de nature courante, ou de routine (comme la délivrance de permis), faisant partie de ses fonctions officielles. Toutefois, afin de laisser aux entreprises le temps d'ajuster leurs pratiques et procédures, cette dernière modification n'est entrée en vigueur que le 31 octobre 2017.

L'amendement de 2013 a en outre étendu considérablement sa portée territoriale en remplaçant la norme de « juridiction territoriale» par une « juridiction de nationalité » ou une compétence dite "personnelle active » (Blyschak, 2014). Cette compétence est fondée sur la nationalité des délinquants, quel que soit l'endroit du monde où leurs actes sont accomplis. Cet amendement permet au Canada de poursuivre une société ou un citoyen canadien pour des actes commis sur un territoire étranger. Il n'est plus obligatoire de prouver l'existence d'un «lien réel et important» entre l'infraction et le Canada pour exercer cette compétence. Le Canada peut aussi, par sa souveraineté territoriale, régir le comportement d'entreprises étrangères, soit celles qui ne possèdent pas sa nationalité ou ne relèvent pas de sa législation, mais qui se livrent à des pratiques de corruption non seulement sur son territoire, mais encore à l'étranger, du moment qu'un des maillons de la chaine des actes constitutifs de corruption prend place sur son territoire ou qu'il existe un lien de rattachement quelconque. Il en va, par exemple, lorsque s'y est tenue une réunion d'un organe de direction de l'entreprise corruptrice au cours de laquelle a été discuté et décidé le stratagème de corruption envisagé à l'étranger; ou encore lorsque des sommes d'argent ayant servi aux actes de corruption ont transité dans un compte tenu dans une banque nationale ou une succursale d'une banque étrangère sise sur son territoire.

\subsubsection{Code criminel}

Afin de connaittre dans quelles mesures et circonstances une organisation canadienne (personnes morales, sociétés, sociétés de personnes, associations, syndicats, municipalités, entreprises à propriété individuelle, etc.) peut être reconnue criminellement responsable des actions ou inactions d'un ou de plusieurs de ses agents, il est indispensable de faire référence au Code criminel L.R.C. [1985], ch. C-46. Depuis l'adoption du projet de loi C-45 en 2003, le Code criminel prévoit des mesures plus répressives concernant la responsabilité criminelle des organisations pour des infractions qui reposent sur la mens rea (c'està-dire pour des infractions qui demandent la preuve d'une intention coupable) comme c'est le cas pour la corruption en vertu de la LCAPE. Avant 2003, une 
organisation ne pouvait être poursuivie, selon la common law, que si un de ses hauts placés (c'est-à-dire l'âme dirigeante de l'entité) avait commis l'infraction, ce qui était, dans les faits, très difficile à démontrer. Désormais, le nouvel article 22.2 du Code criminel prévoit qu'un cadre supérieur ${ }^{6}$, et même un cadre intermédiaire selon l'interprétation des tribunaux ${ }^{7}$, peut engager de trois façons la responsabilité criminelle de son organisation en matière de corruption. Cela sera le cas lorsque dans l'exercice de ses attributions : 1) le cadre participe directement à des pratiques de corruption comme verser un pot-de-vin à un fonctionnaire étranger; 2) le cadre fait en sorte, avec l'intention requise, qu'un autre agent de l'organisation (p. ex., administrateur, associé, employé, membre, mandataire ou entrepreneur) s'engage dans des pratiques de corruption; 3) sachant qu'un autre agent de l'organisation participe à des pratiques de corruption, le cadre omet de prendre les mesures voulues ou raisonnables pour l'en empêcher.

Pour plusieurs observateurs, la relation entre la LCAPE et le Code criminel dans ce dernier cas de figure est plus complexe, plus ambigüe, car l'organisation pourrait être tenue criminellement responsable lorsqu'un de ses cadres est impliqué passivement (il sait, mais ne prend aucune mesure) dans une pratique de corruption internationale commise par un représentant de l'organisation pouvant être un simple employé. Sur un plan pratique, plusieurs questions importantes se présentent (Blyschak, 2014). On peut concevoir qu'il existe des chaînes organisationnelles de fort différentes tailles, et des degrés de diffusion des connaissances afférentes aux problématiques de corruption internationale. Comment élaborer un système de preuve en cette matière? Jusqu'à quel point l'acte de corruption par un agent de l'organisation doit-il être raisonnablement prévisible ou connaissable par un cadre d'un niveau particulier? Quel est le niveau de diligence et de surveillance raisonnable requis dans une organisation pour éviter ce type de situation? Ces questions d'opérationnalisation de l'article 22 c) se posent de façon encore plus exigeante pour les actes de corruption internationale perpétrés par des consultants étrangers agissant au nom d'une société dans le cadre du développement des affaires ou des opérations :

L'utilisation d'agents tiers est souvent inévitable dans les transactions commerciales internationales. Tout d'abord, de nombreux pays étrangers requièrent l'engagement d'un agent local ou d'un parrain local en vertu de la loi. Deuxièmement, même lorsque ce n'est pas une exigence, une représentation étrangère est souvent requise afin de combler les lacunes dans les capacités d'agir d'une société sur le plan international, notamment en fournissant une compréhension approfondie du marché local et de la culture d'affaires, voire des liens et des contacts avec les entreprises pertinentes et des gens d'affaires. Les agents étrangers peuvent également offrir une expertise précieuse dans le contact des bureaucraties locales, sur les obstacles réglementaires et les relations avec les ententes gouvernementales en général [notre traduction]) (Blyschak, 2014, p. 672-673).

En effet, comment les cadres d'une organisation canadienne peuvent-ils s'assurer que les consultants étrangers dans leur travail de démarchage respectent les normes anticorruption fixées par la LCAPE? À quel moment une société canadienne et ses cadres deviennent-ils complices d'une pratique de corruption des consultants étrangers? Même s'il n'y a encore aucune décision des tribunaux canadiens traitant précisément de cette question, celleci pourrait, selon certains observateurs, être balisée en partie par la doctrine de « l'aveuglement ou de l'ignorance volontaire ». Cette doctrine impute une connaissance pénale à une personne «qui a eu des soupçons, a réalisé la probabilité de l'existence d'un fait ou d'une circonstance, mais a préféré ne pas obtenir une confirmation pour pouvoir par la suite en nier la connaissance » (Côté-Harper, Rainville et Turgeon, 1998, p. 388). Appliquée au contexte de la LCAPE, cette doctrine aurait les exigences de connaissance suivantes :

Afin d'éviter la responsabilité criminelle en vertu de la LCAPE pour les actions entreprises par un agent tiers, les sociétés canadiennes et leurs dirigeants devraient veiller à toujours faire preuve de diligence raisonnable à l'égard de tous les agents tiers envisagés pour un mandat particulier, que ce soit en rapport avec le développement des affaires ou autrement, pour déterminer dans quelle mesure les pratiques de corruption peuvent présenter un risque; et ils doivent prendre des mesures préventives pour atténuer le risque de pratiques de corruption si un tel risque a été identifié [notre traduction] (Blyschak, 2014, p. 679). 
Le problème pratique véritable est que les grandes entreprises internationales sont bien souvent réparties sur plusieurs continents, avec moult filiales et établissements, et des centaines, voire des milliers d'employés-cadres ou salariés. Comment, dans ces conditions, assurer la surveillance d'un tel groupe dont l'activité est ainsi déployée et accomplie par tant de personnes? Chaque cas en étant un d'espèce, la diligence raisonnable devra s'apprécier suivant les circonstances.

Il importe de souligner que le suivi des affaires liées à la LCAPE est normalement effectué par des services spéciaux de la police fédérale du Centre de coordination fédérale (CCF) de la Gendarmerie royale du Canada (GRC). Le CCF a le pouvoir de déposer une dénonciation, de faire enquête sur une allégation d'infraction à la LCAPE, de prêter assistance à des demandes d'entraide internationale, d'élaborer et de mettre en œuvre des activités de sensibilisation destinées à des groupes cibles (Gouvernement du Canada, 2017).

Par contre, les sanctions encourues au Canada, même après les importantes modifications de la LCAPE en 2013 et du Code criminel en 2004, semblent plus rigoureuses sur le papier, autant pour les personnes physiques qui les posent que pour les entreprises dont elles relèvent ou qui doivent assumer ces actes. Les cas réels d'enquête, de poursuites et de condamnation se font encore rares. Depuis l'adoption de la LCAPE, trois poursuites ont donné lieu à des plaidoyers de culpabilitép; une poursuite a fait l'objet d'un procès ${ }^{10}$; quatre affaires ayant donné lieu à des accusations n'ont pas encore été tranchées ${ }^{11}$. On constate que très peu de dossiers ont été, dans les faits, véritablement examinés sur le fond par les tribunaux. Pour éviter une mauvaise publicité et les frais d'un long procès, les sociétés préfèrent régler rapidement et déposer un plaidoyer de culpabilité ${ }^{12}$.

À cet effet, le Canada a adopté, en avril 2018, le projet de loi C-74 afin d'intégrer au Code criminel (art. 715.3 à 715.43) un nouvel outil appelé «Accord de réparation $»^{13}$. Il s'agit de la version canadienne du régime d'Accord de poursuite différée (APD) ou Deferred prosecution agreement (DPA) utilisé dans les législations anticorruption aux États-Unis (1992) et au Royaume-Uni (2014) comme solution de règlement pour une société fai- sant l'objet d'une enquête criminelle ou pénale. Selon certains observateurs, ce nouvel outil de justice alternative offre une grande flexibilité pour enquêter et résoudre certains crimes économiques comme la corruption des agents publics étrangers. Les procureurs peuvent ainsi prendre en compte un certain nombre de facteurs dont l'intérêt public, négocier un accord et régler ce litige de manière plus efficace et équitable que ne le ferait un procès traditionnel souvent long et coûteux. Dans tous les cas, l'accord « doit inclure une admission des faits entourant l'infraction, avec un aveu de culpabilité ». L'organisation accepte de payer une pénalité monétaire, renonce au délai de prescription et à respecter certains engagements de conformité en contrepartie de l'extinction des poursuites à son encontre. Au Canada, un tel accord «serait également soumis à l'approbation du tribunal, et une fois approuvé, doit être rendu public» (Dattu, Ritchie et Pavic, 2018).

\subsection{Le droit québécois}

Sur le plan pratique, il semble y avoir un consensus sur le fait que la lutte contre la corruption internationale et nationale est une tâche trop importante pour être satisfaite par le seul droit pénal et que d'autres branches du droit doivent également y contribuer (Bonell et Meyer, 2015). Au Québec, une poursuite ou une condamnation en vertu de la LCAPE, du Code criminel ou de la Loi sur la concurrence, peut avoir des conséquences commerciales importantes pour une entreprise qui désire conclure avec un organisme public québécois tout contrat de construction, de services ou de partenariat public-privé comportant une dépense égale ou supérieure aux montants déterminés par le gouvernement. Depuis 2012, la Loi sur les contrats des organismes publics, L.R.Q., ch. C-65.1 (modifiée par la Loi sur l'intégrité en matière de contrats publics, L.Q. 2012, c. 25) oblige d'une entreprise qu'elle réponde aux "exigences élevées d'intégrité auxquelles le public est en droit de s'attendre d'une partie à un contrat public ou à un sous-contrat public $»^{14}$. Cette exigence d'intégrité vaut aussi pour ses administrateurs, associés, dirigeants ou actionnaires et celle des autres personnes ou entités qui en ont, directement ou indirectement, le contrôle juridique ou de facto. Sur le plan concret, une entreprise doit d'abord obtenir de l'Autorité des marchés financiers (et dans quelques mois de l'Autorité des marchés publics ${ }^{15}$ ) une autorisation 
de contracter et être enregistrée pour une durée de trois ans au Registre des entreprises autorisées (REA). Cette autorisation est renouvelable ${ }^{16}$. L'Autorité des marchés financiers dispose d'un large pouvoir discrétionnaire pour accorder ou refuser cette autorisation. Elle peut prendre en considération le fait qu'une entreprise (ou les autres personnes ou entités mentionnées plus haut) a été poursuivie ou déclarée coupable par un tribunal canadien ou étranger, au cours des cinq années précédentes d'une infraction à la LCAPE ou d'une autre loi pénale (art. 21.38). Dans le cas d'un refus de l'Autorité des marchées financiers d'émettre cette autorisation de contracter, l'entreprise est enregistrée au Registre des entreprises non admissibles aux contrats publics (RENA) ${ }^{17}$. Dans un tel cas, une entreprise ne peut plus contracter avec l'État pendant une période d'au moins cinq ans. Se retrouver ainsi sur le banc de punition peut être très dévastateur pour sa santé financière et sa pérennité (Gosselin et Pellerin, 2013).

Sur le plan individuel, lorsqu'un gestionnaire est membre d'un ordre professionnel (avocat, comptable professionnel, ingénieur, administrateur agréé, etc.), une condamnation en vertu de la LCAPE peut avoir des conséquences très graves sur la carrière. Le Code des professions, L.R.Q., c. C-26, prévoit spécifiquement, depuis 2013, que le fait pour un professionnel de commettre, de tenter de commettre un acte impliquant de la collusion, de la corruption, de la malversation, de l'abus de confiance ou du trafic d'influence ou de comploter en vue de la commission d'un tel acte, est un acte dérogatoire à la dignité de sa profession (art. 59.1.1.). En juin 2017, l'adoption de la Loi modifiant diverses lois concernant principalement l'admission aux professions et la gouvernance du système professionnel18 va encore plus loin en obligeant chaque ordre professionnel à inclure dans son code de déontologie " des dispositions énonçant expressément qu'est interdit tout acte impliquant de la collusion, de la corruption, de la malversation, de l'abus de confiance ou du trafic d'influence» et «des dispositions obligeant le membre d'un ordre à informer le syndic lorsqu'il a des raisons de croire qu'une situation susceptible de porter atteinte à la compétence ou à l'intégrité d'un autre membre de l'ordre survient» (art. 51). Les sanctions qui seront imposées peuvent aller jusqu'à la radiation permanente ou la révocation du permis d'exercice du professionnel.

\section{Les programmes de sensibilisation, les outils de soft law et les systèmes de management anticorruption}

Au-delà de ses lacunes et de ses zones d'ombre, le droit national et international n'aurait pas l'efficacité et l'impact voulus pour prévenir et intervenir en matière de corruption dans les transactions commerciales internationales, s'il n'était pas soutenu par des outils de soft law (droit mou) tels des codes de conduite ${ }^{19}$, des programmes de sensibilisation ou encore des systèmes de management anticorruption au sein même des entreprises. Les normes internationales et nationales (même les plus parfaites) doivent être appropriées au sein de la culture des organisations, et au final dans le comportement éthique des individus, pour voir les consciences et les comportements se modifier dans le court terme. Selon une étude empirique de Köbis et al., (2015), les croyances sur la prévalence de la corruption dans un contexte spécifique font reproduire les comportements corrompus. Les résultats de l'enquête démontrent en effet que les normes descriptives perçues peuvent influer sur le niveau de comportement corrompu et pourraient offrir une explication de la variation interpersonnelle et interculturelle du comportement corrompu dans le monde réel. Dans des contextes sociaux hautement corrompus, le « ciment des normes sociales » stabilise la corruption tandis que dans un contexte de corruption faible, il fait le contraire : permettre à la non-corruption d'être la "chose normale à faire » (Köbis et al., 2015).

\subsection{Les codes de conduite anticorruption adoptés par les entreprises}

De nombreuses entreprises ou organisations exerçant leur activité à l'échelle mondiale ont adopté de leur propre initiative un code de conduite (souvent appelé code de bonne conduite, code d'éthique, code de déontologie, charte, etc.) consistant en un engagement «en matière de bonnes pratiques de gestion» à se conformer à certaines valeurs et normes de comportement dans leurs activités ou opérations (Desbarats, 2003) ${ }^{20}$. Par valeurs, on doit entendre les grands principes (p. ex., intégrité, engagement vers l'excellence, etc.) qui sous-tendent les comportements que l'entreprise entend privilégier dans le cadre de ses opérations. Les comporte- 
ments souhaités se matérialisent dans une énumération de pratiques devant être adoptées à l'égard de certaines situations problématiques dans des domaines aussi variés que la gestion de l'environnement, les conditions de travail, la diffusion d'information, la concurrence, la protection du consommateur, etc. (Benyekhlef, 2016).

De façon spécifique, les codes de conduite des entreprises contiennent fréquemment des instructions sur la corruption internationale, et ce, à l'intention de leurs dirigeants, cadres, employés et partenaires contractuels. Elles visent à prévenir et détecter les cas de corruption (privée et publique) quelles que soient les pratiques et coutumes locales dans les territoires où l'entreprise exerce ses activités. Elles précisent les comportements admis (p. ex., acceptation de cadeaux d'une valeur raisonnable) et ceux qu'il convient d'éviter (p. ex., paiements illicites, contributions caritatives, etc. ${ }^{21}$ ), autant dans les relations nouées avec des clients et fournisseurs privés que dans le cadre des processus menant à l'octroi de contrats ou marchés publics, et donc au regard des relations avec des agents publics $^{22}$. Certaines entreprises vont produire un code particulier sur cette dernière question, vu son importance. Une grande entreprise multinationale pourra même adopter plusieurs codes sur ce thème, afin de traiter très spécifiquement d'États présentant des risques particuliers.

Selon certaines études, pour être efficaces, les codes de conduite doivent énoncer clairement les règles et lignes directrices dont l'entreprise souhaite l'application.

The literature agrees on the fact that corruption may be the result of lacking or ineffective guidelines. Companies are more vulnerable to corruption when they have no policies to guide them in such situations (Lane and Simpson, 1984) and when individuals feel ambiguity about what the expectations of behavior are in these situations (Litzky et al., 2006). Despite few exceptions, the reviews of Ford and Richardson (1994), Loe and his colleagues (2000), and O'Fallon and Butterfield (2005) showed that the majority of studies support the idea that the existence of a code of ethics is positively related to ethical decision making raising the general level of awareness of ethical issues (Rabl, 2011, p. 89).
Par conséquent, les dirigeants, les cadres, les employés, les partenaires contractuels étrangers, y compris les représentants (agents) indépendants devront pouvoir saisir la manière de se comporter lorsqu'il se présente des situations signalant des pratiques de corruption. Le code de conduite désignera les responsables ou les membres des services juridiques ou éthiques de l'entreprise à qui ces personnes devront s'adresser afin de s'assurer de respecter les politiques de l'entreprise. Un lanceur d'alerte (whistleblower) y décèlera, pour sa part, les moyens de signaler toute pratique contraire aux lois anticorruption ou au code de conduite.

Le code de conduite de l'entreprise s'applique normalement à tous les dirigeants, cadres, employés et même aux partenaires contractuels étrangers, y compris les représentants (agents) indépendants de l'entreprise, du fait qu'un acte de corruption posé par l'un d'entre eux sera généralement attribué à l'entreprise dont ils relèvent ou qui leur a accordé un contrat (dans le cas des intermédiaires indépendants), s'il a prétendu agir au nom de celle-ci. Le code devra donc être annexé, en tant que partie intégrante, autant aux contrats d'emploi qu'à ceux qui impliquent les partenaires contractuels étrangers. Par ailleurs, ainsi que le suggère le Guide de bonnes pratiques pour les contrôles internes, la déontologie et la conformité de l'OCDE (2010), le code s'appliquera aussi à toutes les entités sur lesquelles l'entreprise exerce un contrôle effectif par des liens en capital, et tout spécialement les filiales. Mais ce contrôle peut aussi provenir de rapports simplement contractuels, lorsqu'une dépendance de fait est créée chez l'un des partenaires à l'égard de l'autre. L'on peut songer ici aux contrats de concession, de franchise, et, plus largement, de transfert de droits de propriété industrielle (brevets d'invention, marques et logos, dessins industriels).

Plutôt que d'inclure le code de conduite de l'entreprise en annexe des contrats qu'elle conclut avec ses partenaires d'affaires étrangers, il peut être stipulé que ces demiers doivent, s'ils n'en ont pas déjà, se doter d'une politique anticorruption propre, d'un niveau élevé, assortie d'un mécanisme d'application efficace faisant l'objet de contrôles réguliers et indépendants (monitoring). Il doit s'agir d'un processus de contrôle continu, qui doit opérer d'une façon d'autant plus minutieuse que l'État où s'accomplissent les prestations contractuelles est perçu comme étant particulièrement corrompu, que le contrat est important et que le 
partenaire contractuel n'apparaît pas d'une frabilité à toute épreuve sur le plan de ses pratiques anticorruption. En ce sens, une disposition peut prévoir un audit périodique des mécanismes institués par le partenaire, qui peut même comprendre des inspections, selon des modalités à préciser dans le contrat. Néanmoins, on peut concevoir qu'un tel contrôle sera difficile à exercer en pratique lorsque la «chaîne de valeur» ajoutée de l'entreprise est dispersée sur le plan géographique, au surplus parmi plusieurs partenaires contractuels. L'on peut s'attendre toutefois à ce que le contrôle soit exercé sur les plus proches partenaires, et moins à l'égard des sous-traitants auxquels ceux-ci ont pu confier l'exécution de certaines prestations.

\subsection{Modèles de codes de conduite sectoriels proposés par des institutions privées et des ONG}

Parallèlement aux initiatives des entreprises ellesmêmes, certaines associations et catégories professionnelles proposent des codes de conduite à l'intention de l'ensemble de leur secteur d'activité, voire des modèles dont peuvent s'inspirer leurs membres au moment de la conception de leur propre code.

Ainsi, la Chambre de commerce internationale (CCI ou ICC en anglais), qui représente mondialement les entreprises et a pour objectif de favoriser les échanges et l'investissement, l'ouverture des marchés aux biens et aux services, et la libre circulation des capitaux, entend jouer un rôle de leader dans la lutte contre la corruption ${ }^{23}$. Elle met l'accent sur la nécessité, pour les entreprises, d'instituer et, le cas échéant, d'accroître l'efficacité de leurs programmes d'autorégulation portant sur la corruption.

Les réalisations de la CCI sur le plan de la lutte anticorruption sont contenues dans plusieurs instruments qu'elle a conçus progressivement au cours des années. Il y a d'abord les Règles d'ICC pour combattre la corruption - ICC Rules on Combating Corruption (Commission d'ICC sur la responsabilité sociale de l'entreprise et la lutte contre la corruption, 2011). Émises pour la première fois en 1977, ces règles d'autodiscipline forment un ensemble de lignes directrices visant la promotion de standards élevés de conduite de la part des sociétés. Le 26 mars 1996, le comité exécutif a adopté une mise à jour des règles et a étendu leur champ d'application matériel. Si, en 1977, les règles prohibaient seulement l'extorsion et la corruption afin d'obtenir ou de maintenir des affaires, les nouvelles règles les prohibent à toutes fins. Elles furent révisées à nouveau en 1999, 2005 et 2011, en sorte qu'elles couvrent aujourd'hui des questions plus générales, comme les cadeaux et échanges de faveurs entre partenaires d'affaires, les contributions au financement d'organisations politiques ou aux comptes de campagne de candidats à des fonctions électives ${ }^{24}$. Elles concernent la corruption d'agents publics et privés. L'approche de base des Règles d'ICC pour combattre la corruption est que la lutte contre la corruption nécessite l'action de tous les intervenants, qu'il s'agisse des entreprises, des gouvernements et des organisations internationales, gouvernementales ou pas. L'objectif ultime est l'atteinte d'une plus grande transparence dans les transactions commerciales internationales. La CCI a aussi émis des lignes directrices concernant des volets particuliers du thème de l'intégrité ${ }^{5}$. Elle a même conçu une clause anticorruption (ICC Anti-corruption Clause) pouvant être incluse dans n'importe quel type de contrats de commerce international (International Chamber of Commerce, 2012) ${ }^{26}$.

\subsection{Politique de gestion du risque et mécanismes de dénonciation ou signalement des irrégularités}

Les règles contenues dans les codes de conduite ont pour principal objectif de créer une culture anticorruption au sein de l'entreprise. Mais il est vital qu'elles soient appliquées dans les faits. Généralement, une entreprise conçoit de façon parallèle une politique de gestion du risque de corruption par l'adoption d'une approche autant préventive, afin d'éviter que des cas ne se produisent, que réactive, à la suite de la découverte d'un cas avéré. On aura, par exemple, intérêt à se doter d'un système de management anticorruption, en particulier celui proposé en 2016 par l'Organisation internationale de normalisation (ISO) avec la norme ISO 37001 et de le faire certifier par une tierce partie. C'est ainsi qu'une entreprise se dote souvent de mécanismes de détection, d'enquête et de sanction des faits de corruption pouvant survenir dans l'exercice de son activité, en son sein ou du fait de ses partenaires contractuels étrangers. 
On retrouve, à cet effet, dans la Convention des Nations Unies contre la corruption une demande aux États participants d'envisager la mise en place de mesures et de systèmes de nature à faciliter le signalement, par les agents publics, aux autorités compétentes, des actes de corruption dont ils ont connaissance dans l'exercice de leurs fonctions (art. 8, par. 4). Elle demande aussi d'envisager l'incorporation, dans leur système juridique interne, de mesures appropriées pour assurer la protection contre le traitement injustifié dont pourrait faire l'objet toute personne qui signale aux autorités compétentes, de bonne foi et sur la base de soupçons raisonnables, tous faits concernant les infractions établies conformément à la convention (art. 33).

Toute entreprise se trouve encouragée à se doter d'un mécanisme de dénonciation (whistleblowing), c'est-à-dire devant permettre aux personnes qui possèdent des informations de première main à propos de situations pouvant être qualifiées de pratiques de corruption, de les dénoncer sans qu'elles puissent encourir de représailles pour avoir ainsi agi, dans le meilleur intérêt de leur commettant. Il s'agira, par exemple, de la mise à disposition de lignes téléphoniques, ou autres moyens, qui permettront à tous ceux qui ont des raisons sérieuses de croire que des actes de corruption ont été posés, de les dénoncer à une personne spécifiquement mandatée sur ce plan. Ils n'auront pas, en conséquence, l'obligation de passer par les canaux habituels de la hiérarchie, susceptibles d'étouffer l'affaire.

Par ailleurs, les cas dénoncés doivent faire l'objet d'un examen et d'une enquête menés avec diligence et professionnalisme. C'est pourquoi le code de conduite, ou un document annexe devra contenir un protocole détaillé d'enquête lorsqu'un acte de corruption est découvert ou qu'une situation donne lieu à des soupçons en raison des «signaux d'alerte» qu'elle émet. Si l'enquête révèle une preuve manifeste d'un acte qui est soit illégal soit contraire aux règles du code, une mesure disciplinaire devra être prise à l'encontre des personnes responsables. Cette sanction ira d'un avertissement, dans le cas d'une infraction mineure, jusqu'au congédiement immédiat s'il s'agit d'une infraction sérieuse. Puis, comme elle doit le faire à la suite de la révélation de chacun des cas, l'entreprise réexaminera ses politiques et procédures afin d'apprécier leur efficacité au regard de la détection hâtive de cas de corruption ou de leur découverte diligente après coup.

La stratégie de la gestion du risque de corruption doit aussi tenir compte de la géographie. Les États développés tendent généralement, de ce point de vue, à être sans danger pour les entreprises, bien que la corruption n'y soit pas absente, comme le démontre avec éloquence le rapport de l'OCDE de 2014 sur la corruption transnationale (OCDE, 2014). En revanche, les États instables sur le plan politique, de même que les États les plus pauvres, qui sont souvent les mêmes, figurent généralement parmi les plus corrompus dans l'Indice de perception de Transparency International (TI). Les activités qui y sont exercées doivent donner lieu à un contrôle beaucoup plus strict. La situation politique, économique et sociale peut cependant évoluer substantiellement durant les années au cours desquelles l'entreprise exerce son activité dans un État. D'où la nécessité de pouvoir réévaluer la situation sur le plan du risque de corruption en cas de changement de circonstances, qu'elles soient favorables ou non.

Il faut tenir compte, à cet égard, du fait que plusieurs éléments de la " chaîne de valeur ajoutée » de l'entreprise mondialisée d'aujourd'hui sont susceptibles d'être dispersés sur le plan géographique. Les marchés qu'elle dessert peuvent l'être aussi. Cette dissémination géographique nécessite souvent qu'elle soit représentée sur place pour la gestion des relations contractuelles avec les fournisseurs et distributeurs étrangers. Les représentants de l'entreprise, s'ils doivent obtenir des autorisations ou permis pour opérer, sont fréquemment tenus, pour ce faire, de verser des paiements de facilitation aux agents publics dans certains États. Ils peuvent aussi être tentés de les proposer même quand ils n'y sont pas tenus, afin d'accélérer les démarches. De tels paiements sont toutefois proscrits par certaines législations, dont celle du Royaume-Uni et, aujourd'hui, du Canada, depuis que celui-ci a modifié, en 2013, sa Loi sur la corruption d'agents publics étrangers.

Une entreprise est donc particulièrement vulnérable à la grande et petite corruption pouvant résulter des actes de ses représentants à l'étranger, 
même si son activité se déploie dans les domaines considérés comme n'étant pas particulièrement à risque. En somme, lorsqu'une entreprise exerce une activité à l'étranger, même de faible ampleur, et qu'elle agit par l'intermédiaire d'un représentant indépendant, elle doit prévenir celui-ci des conséquences de ses gestes et l'«inviter», en conséquence, à s'engager au respect de ses politiques anticorruption, si l'agent n'en a pas déjà une de qualité équivalente.

Les mécanismes de contrôle, lorsqu'ils sont correctement appliqués, sont donc fondamentaux. Ils permettront à l'entreprise d'opposer, le cas échéant, une défense valable de diligence raisonnable devant les tribunaux en cas de poursuite, en démontrant qu'elle a pris tous les moyens en son pouvoir afin de prévenir la commission d'actes de corruption. L'action préventive de l'entreprise lui permettra de négocier avec les autorités de l'État un règlement plus avantageux qu'en l'absence d'un tel mécanisme, dans l'éventualité où l'affaire ne se rendrait pas jusqu'au prononcé du jugement. En outre, sa réputation en souffrira moins aux yeux de ses "parties prenantes ». Par ailleurs, elle pourra éviter de se voir exclue de l'attribution de marchés publics, notamment ceux financés par les agences de développement de l'État, de la Banque mondiale et des banques régionales de développement, ou encore par les institutions de l'Union européenne (UE) ou de ses États membres.

\subsection{Engagement (leadership) des dirigeants dans la prévention et la détection des faits de corruption}

Au-delà des mécanismes institutionnels formels, plusieurs études en éthique appliquée ont démontré l'importance de l'engagement des dirigeants et des cadres afin d'édifier une culture organisationnelle axée sur l'honnêteté, l'équité, le respect, la responsabilité et la transparence.

Les dirigeants aiment souvent communiquer leur philosophie et les valeurs qui leur tiennent à coeur au cours des rencontres avec leurs employés ou dans les vidéos qui sont destinées à la socialisation des nouveaux arrivants (Schein, 1985). Toutefois, leur comportement au quotidien, de même que celui de leurs gestionnaires, permet de découvrir les pratiques acceptables et les façons de faire encouragées ou inacceptables, et ce, bien plus efficacement que les énoncés de valeurs formels, les codes de conduite, les procédures et les règles. Les valeurs perçues comme étant privilégiées dans leurs décisions et leurs actions donnent le ton et servent de guide aux autres acteurs organisationnels (Girard, 2009, p. 104; Treviño et al., 1999; Jose et Thibodeaux, 1999; Anand, Ashforth et Joshi, 2005).

En ce sens, un programme anticorruption efficace dans une organisation nécessite, au-delà du discours officiel, une implication réelle de la haute direction. Sur le plan concret, les dirigeants et les cadres ne doivent pas tolérer, même implicitement, «l'utilisation de rationalisations pour justifier certains gestes discutables » (Girard, 2007, p. 104). Un défaut d'engagement soutenu et visible de celle-ci est presque toujours fatal à son succès. Il faut, à cet égard, signaler que, dans la majorité des affaires examinées dans le rapport de l'OCDE sur la corruption transnationale, les membres de la direction de l'entreprise (41\%), voire le président-directeur général $(12 \%)$, étaient informés des actes de corruption s'étant produits ou les avaient approuvés (OCDE, 2014).

La haute direction doit, entre autres, démontrer son adhésion à la lutte anticorruption par la dotation de ressources appropriées. Un programme anticorruption efficace peut en effet nécessiter d'importantes ressources financières et humaines. Il sera parfois requis, par exemple, de former une équipe de contrôle multidisciplinaire lorsque l'appui et l'engagement de l'entreprise tout entière sont recherchés. En outre, un engagement résolu de tout le monde au sein de l'entreprise est primordial. L'implication des cadres intermédiaires et salariés dès les premiers stades de son élaboration accroîtra les possibilités que le programme fasse l'objet d'une application réelle vu l'appui reçu à leur niveau, tout en lui permettant d'atteindre plus rapidement son stade de maturité lors de sa mise en œuvre.

\subsection{Programme de communication et de formation}

La communication et la formation sont aussi essentielles au succès du programme anticorruption, de sorte que tous, dans l'entreprise, le connaissent, le 
comprennent et soient en mesure de l'appliquer lorsque surgissent des cas concrets. Le code de conduite devra faire l'objet d'une ample diffusion de façon à ce que chacun puisse en prendre connaissance. Son existence et son contenu devront, en conséquence, être rappelés fréquemment à tous ceux auxquels il s'applique, avec une attention toute particulière à l'endroit des cadres et salariés nouvellement embauchés.

La communication devra être complétée par des formations comportant un volet pratique substantiel avec, idéalement, la simulation de circonstances réelles. Les formations portent, notamment, sur l'identification de « drapeaux rouges » (red flags), des signaux d'alerte révélateurs d'actes de corruption à l'intérieur d'une entreprise, ou encore chez les partenaires contractuels étrangers, y compris les représentants indépendants. Ils consistent, en général, en des comportements ou événements inhabituels, qui éveillent la suspicion chez une personne raisonnable.

À cet effet, la Chambre de commerce internationale (CCI) propose un outil de formation des cadres et salariés basé sur 22 scénarios réels et appelés RESIST (Résister aux extorsions de fonds et aux sollicitations dans le cadre des transactions internationales). Il doit être utilisé conjointement avec deux guides pratiques : ICC Ethics and Compliance Training Handbook (Vincke et Kassum, 2013) et Fighting Corruption - International Corporate Integrity Handbook (Heimann et Vincke, 2008). Ce dernier fut rédigé à l'intention des entreprises afin qu'elles puissent se conformer aux règles et à la convention de l'OCDE de 1997 sur la lutte contre la corruption d'agents publics étrangers dans les transactions commerciales internationales.

\subsection{Rapport annuel (reporting)}

L'entreprise peut rendre compte, dans la partie de son rapport annuel portant sur sa responsabilité sociale et environnementale, de son action à l'encontre de la corruption. Bien sûr, rien ne l'y oblige pour le moment, y compris au Canada. Mais il est envisageable qu'une telle obligation leur soit imposée éventuellement par les législateurs nationaux (ou par l'Union européenne), du moins ceux des États développés. Quoi qu'il en soit, que la divulgation de ses initiatives en matière de lutte contre la corruption soit spontanée ou résulte d'une obligation, l'entreprise pourra s'inspirer de référentiels de publication d'informations environnementales et sociétales proposés par de nombreux organismes. C'est notamment le cas de la $4^{\mathrm{e}}$ version en 2013 des Lignes directrices pour le reporting développement durable (G4) conçues par l'ONG Global Reporting Initiative (GRI).

Le reporting développement durable aide les organisations à définir leurs objectifs, à mesurer la performance et à gérer le changement afin d'accroître la durabilité de leurs opérations. Un rapport de développement durable transmet les Éléments d'information sur les impacts d'une organisation - qu'ils soient positifs ou négatifs sur l'environnement, la société et l'économie (Global Reporting Initiative, 2013, p. 3).

Sur le plan de la sous-catégorie de la dimension « société » du rapport figure la lutte contre la corruption. À ce sujet, les lignes directrices du GRI-G4 ont trait au dévoilement (reporting) des trois aspects précis suivants : le nombre total et le pourcentage des sites stratégiques qui ont fait l'objet d'une évaluation des risques de corruption et les risques substantiels identifiés (G4-S03); la communication et la formation des membres des instances de gouvernance, des salariés, des partenaires commerciaux sur les politiques et procédures de l'organisation en matière de lutte contre la corruption (G4-S04); et enfin l'indication des cas avérés de corruption et les mesures prises (G4-S05).

\subsection{Pactes d'intégrité ou déclaration concernant la corruption}

Les pactes d'intégrité sont des instruments visant à améliorer les rapports de confiance entre les partenaires commerciaux. C'est un engagement actif des organisations, des entreprises, en coopération avec la société civile, de faire la promotion des principes universels des droits de l'homme, des relations de travail, de la protection de l'environnement et de lutte contre la corruption.

\subsubsection{Pacte mondial des Nations Unies}

Le dixième principe du Pacte mondial (Global Compact) des Nations Unies fait directement référence à la lutte contre la corruption (ONU, 2000). L'idée d'un Pacte mondial avec les entreprises multinationales fut lancée à Davos en 1999 par le Secrétaire général de l'ONU de l'époque, Kofi Annan. En permettant aux sociétés multinationales de devenir des partenaires à part entière des Nations Unies, on déroge du principe qui veut que seuls les États soient des partenaires de l'ONU. Le Pacte mondial est en quelque sorte un «contrat d'adhésion dont les termes sont fixés par 
l'ONU », qui permet aux entreprises d'adhérer spontanément « aux principes juridiques qui lient déjà les États » (Decaux, de Frouville, 2012, p. 506). Parmi ces principes, plusieurs sont déjà consacrés par les États dans des instruments internationaux, notamment la Déclaration universelle des droits de l'homme, la Déclaration de l'OIT sur les principes et droits fondamentaux de l'environnement, les principes de Rio sur l'environnement et le développement et la Convention des Nations Unies contre la corruption de 2003. En adhérant à ce Pacte mondial, les entreprises sont « invitées à agir contre la corruption sous toutes ses formes, y compris l'extorsion de fonds et les pots-devin $»^{27}$. Bref, avec le Pacte mondial des Nations Unies, on a cherché à dialoguer avec les sociétés transnationales et à les encourager à adopter dans leur gouvernance divers principes universels (Tomasic, 2018). Le Pacte mondial n'est pas contraignant, mais il incorpore un cadre de divulgation obligatoire. Les entreprises doivent donc communiquer, sur une base annuelle, leurs progrès à leurs parties prenantes et les afficher sur le site Internet de l'ONU Global Compact. À défaut de le faire, durant deux années consécutives, une entreprise se verra expulsée du Pacte mondial (ONU, 2000) ${ }^{28}$.

\subsubsection{Pactes d'intégrité de Transparency International}

Transparency International (TI) met à la disposition des entreprises (et aussi des agents publics) des "pactes d'intégrité (PI)» dans certains domaines, comme les services publics économiques (énergie, assainissement des eaux, matières résiduelles ${ }^{29}$. Créés dans les années 1990, ces PI prévoient, par exemple, que les entreprises concurrentes pour l'obtention de marchés publics dans ces secteurs d'activité peuvent s'engager entre elles à ne pas verser de pots-de-vin; et, réciproquement, de la part des membres des commissions de marchés, s'obliger à ne pas en recevoir.

Ces PI ont pour caractéristique de permettre l'implication d'un tiers indépendant ou d'une ONG, telle TI, dans le processus d'attribution des marchés. Ils offrent aussi la possibilité d'instituer des mécanismes préventifs, notamment par l'invitation adressée aux personnes siégeant à la commission des marchés à faire une déclaration de patrimoine, avant et après l'attribution du contrat. Par ailleurs, au terme de l'appel d'offres, les entreprises concurrentes s'engagent à publier les frais connexes à la préparation de leur soumission. En ce sens, le PI stipule que les soumissionnaires doivent dévoiler toutes les sommes qu'ils ont versées dans le cadre du processus d'attribution d'un marché.

Des sanctions s'appliquent en cas de violation du PI. Elles comprennent notamment la perte ou le refus du marché, l'obligation d'octroyer des dommagesintérêts, l'inscription sur une « liste noire » relativement aux futurs appels d'offres et enfin, des sanctions disciplinaires ou pénales à l'endroit des agents publics fautifs, membres des commissions de marchés. L'existence de tels dispositifs a pour conséquence de supprimer toute justification de la part des entreprises qui font fi de leurs dispositions, lorsque des comportements de corruption trouvent leur origine en leur sein ou proviennent d'actes posés par leurs partenaires contractuels étrangers.

\section{Conclusion}

Au cours des deux dernières décennies, l'expansion de l'activité des entreprises à l'échelle mondiale a obligé de nombreux États, dont le Canada, à s'engager plus activement et efficacement afin de prévenir, détecter et punir les faits de corruption. Des conventions internationales ont été signées, des lois nationales anticorruption ont été adoptées, des guides de bonnes pratiques ont été créés à cet effet, ce qui est certes un pas dans la bonne direction. Cependant, plusieurs observateurs ont constaté qu'il subsiste encore beaucoup de résistance à la mise en application de ces lois (Heimann, Földes et Coles, 2015). Et parmi les 41 pays signataires de la Convention sur la lutte contre la corruption de l'OCDE, le Canada n'apparait pas comme un premier de classe à cet égard. Nous verrons avec le temps si l'adoption du projet de loi C-74 afin d'intégrer au Code criminel canadien le nouveau régime d'Accord de poursuite différée (APD) va lui permettre d'améliorer ses performances.

Par ailleurs, on se doit de constater que les entreprises ou les organisations exerçant leur activité à l'échelle mondiale n'ont plus guère le choix de prendre très sérieusement en considération les risques de corruption, car les coûts pour leurs affaires à la suite de poursuites ou de condamnations peuvent devenir exorbitants. Au moment des visites sur place menées dans le cadre de la phase 2 du mécanisme de suivi de la convention de l'OCDE, le Groupe de travail sur la corruption a d'ailleurs pu observer la propension, parmi les grandes entreprises multinationales, à délaisser les grandes références à l'éthique et à la morale dans 
leurs codes de conduite, au profit de références plus précises aux lois nationales et aux conventions internationales visant à lutter contre la corruption, ainsi qu'au fait que les pots-de-vin versés à des agents publics étrangers ne sont généralement plus déductibles des revenus de l'entreprise (OCDE, 2006). L'on constate aussi une tendance de plus en plus prononcée à l'engagement des instances dirigeantes dans la prévention et la détection des faits de corruption, à l'adoption d'un dispositif d'alerte interne visant à encourager la déclaration des méfaits (whistleblowing), et protéger les lanceurs d'alerte contre les mesures de représailles, à l'instauration de procédures d'évaluation des partenaires contractuels, de dispositifs de formation aux risques de corruption, et d'un autre pour le contrôle et l'évaluation internes (Agence française anticorruption, 2017).

La lutte à ces dérives, que sont la corruption et les pratiques fiscales abusives, semble, lentement mais sûrement, en train de s'intensifier. Les déclarations finales émises par les chefs d'État et de gouvernement au sortir de leurs sommets, qu'ils soient du G8, du G7 (quand la Russie en a été exclue afin de stigmatiser son comportement guerrier en Ukraine) ou du G20, comportent presque toujours la référence aux deux genres de comportements répréhensibles, à l'action résolue exercée à leur encontre, et faisant le point sur les progrès accomplis à leur égard.

On ne peut manquer cependant de rappeler que les acteurs économiques sont de fait amenés à opérer dans une structure mondialisée complexe: cet ensemble d'institutions économiques et sociales leur préexiste, et souvent oblige à agir d'une manière violant les règles formelles de leur activité. La poursuite de la dominance sur un terrain stratégique et de marché se traduit depuis longtemps par des écarts de pratique par rapport aux formulations canoniques de l'économisme standard. Ainsi le marché global est-il susceptible de multiplier les occasions et comportements de corruption, en particulier lorsque des acteurs deviennent extrêmement dominants plutôt que simplement concurrentiels. La hiérarchie sociale des entreprises, une oligarchie, se trouve alors à amplifier, démultiplier les vices personnels, à sélectionner de fait les corrompus pour ce qui est de l'occupation des postes de prestige et surtout de pouvoir dans cette structure.

Le contexte de cette mutation progressive dans la lutte à la corruption est complexe à situer précisément, car il est composé d'un amalgame d'éléments divers, qu'il s'agisse de résoudre les excès du capitalisme financier révélés dans le sillage de la crise de 2008-2009, ou encore l'aggravation du terrorisme international qui suscite des mesures visant à courtcircuiter son financement. Les affaires fortement médiatisées impliquant des entreprises de notoriété mondiale ont mis en lumière l'étendue avec laquelle la corruption dénature les marchés et détruit les communautés nationales au sein desquelles elle prend racine. Des campagnes nationales et internationales déclenchées par les ONG ont relayé les demandes de la société civile en faveur d'une application résolue des lois nationales de mise en œuvre des conventions internationales anticorruption et, au-delà, d'une plus grande transparence de la part de tous les acteurs vulnérables à l'égard d'actes de corruption internationale et d'évasion fiscale.

\section{NOTES}

1 Afin d'apprécier l'étendue de la corruption dans le monde, on consultera utilement les indices suivants : l'indice des payeurs de pots-de-vin (BPI) de la Transparency internationale (Bribe Payers Index); l'Enquête du Système National d'Intégrité (ESNI) Rapport d'évaluation qualitative des piliers d'intégrité; le Rapport et indicateurs qualitatifs sur la transparence dans les secteurs du pétrole, du gaz et des mines, l'Indice Ibrahim de la Gouvernance africaine (IIAG) : l'Indice de gouvernance de la Banque mondiale : l'Indice "Rule of Law" du "World Justice Project" .

2 Foreign Corrupt Practices Act of 1977 (FCPA) (15 U.S.C. $\$ 78 \mathrm{dd}-1$, et seq.).

3 Le Canada a signé cette convention le 21 mai 2004 et l'a ratifiée le 2 octobre 2007.

4 L'Académie internationale de lutte contre la corruption (IACA) contribue à la mise en œuvre de cette convention. «L'UNODC, en sa qualité de gardien de la Convention, fournit une assistance technique aux États parties et aux États signataires en les aidant à assurer l'intégrité de leur système judiciaire, à améliorer leur législation, à échanger les bonnes pratiques et à élaborer des stratégies pour lutter efficacement contre la corruption ». ONU. (n/d).

5 Loi sur la corruption des agents publics étrangers (L. C. 1998, chap. 34); modifiée par (L.C. 2013, chap. 26). La LCAPE est entrée en vigueur le 14 février 1999. 
6 On entend par cadre supérieur, un individu qui joue un rôle important dans la détermination des politiques de la société, gère une partie importante des activités de la société ou encore un administrateur ou le chef de la direction ou le directeur financier de la société (Blyschak, 2014).

7 Dans l'affaire Metron, le tribunal considère que les actions d'un cadre intermédiaire, c'est-à-dire le superviseur d'un chantier de construction d'une petite entreprise de construction de Toronto, peuvent entraîner la responsabilité criminelle de l'organisation. R. c. Metron Construction Corporation, 2012 ONCJ 506, 1 CCEL (4 th) 266; R. v. Metron Construction Corporation, 2013 ONCA 541; R. c. Pétroles Global Inc. 2012 QCCQ 5749, 2015 QCCS 1618.

8 Ainsi, les actions de diligence raisonnable (due diligence), d'atténuation des risques contractuels à l'égard des consultants étrangers, peuvent prendre plusieurs formes : 1) considérer le pays et l'industrie d'où le consultant est engagé; 2) enquêter sur les références, qualifications, antécédents scolaires et professionnels, affiliations du consultant, ainsi que sur son personnel; 3) être vigilant lorsque vient le temps de fixer ses honoraires afin de les comparer avec ceux facturés par d'autres consultants œuvrant dans le même marché. La diligence raisonnable voudrait aussi que le consultant fournisse des garanties et des engagements contractuels concernant les pratiques de corruption et accepte que la société qui l'engage puisse effectuer de façon constante la vérification de ses livres et dossiers (Blyschak, 2014).

9 R. v. Watts, [2005] A.J. No. 568 (Hydro-Kleen); Calgary (Alberta), Her Majesty the Queen v. Niko Resources Ltd., E-File No. : CCQ11NIKORESOURCES, 24 juin 2011; Her Majesty the Queen v. Griffiths Energy International, E-File No. : CCQ13GRIFFITHSENER, Action No. 130057425Q1, 25 janvier 2013.

10 R. v. Karigar 2013 ONSC 5199 confirmé par la Cour d'appel de l’Ontario R. v. Karigar, 2017 ONCA 576.

11 Groupe SNC-Lavalin Inc.; MM. Sami Bebawi, Constantine Kyres et Stéphane Roy; MM. Robert Barra, Dario Berini et Shailesh Govindia; MM. Robert Barra, Dario Berini et Shailesh Govindia; Larry Kushniruk.

12 Les sociétés, contrairement aux particuliers, ne peuvent pas être emprisonnées. Par contre, en raison des fortes peines qu'ils peuvent encourir, les particuliers n'ont souvent pas d'autres choix que de subir un procès pour présenter une défense.

13 Projet de loi C-74, Loi no 1 d'exécution du budget de 2018, déposé le 27 mars 2018. Repéré à http:/ /www.parl.ca/ DocumentViewer/fr/42-1/projet-loi/C-74/sanction-royal

14 Ce montant peut varier selon la catégorie de contrat. Le gouvernement du Québec a prévu une mise en place graduelle par décrets de cette loi. Pour connaitre ces montants, on consultera le site de l'Autorité des marchés financiers à l'adresse suivante : https://www.lautorite.qc.ca/fr/contrats-publics.html

15 L'Autorité des marchés publics (AMP) a été créée en décembre 2017 par la Loi 108, intitulée Loi favorisant la surveillance des contrats des organismes publics et instituant l'Autorité des marchés publics L.Q. 2017, c.17. L'AMP va remplacer à l'automne 2018 l'Autorité des marchés financiers (AMF) dans ses compétences qu'elle avait depuis 2011 à ce sujet.

16 L'Unité permanente anticorruption (UPAC) qui a pour mission d'effectuer les suivis de l'intégrité des entreprises pour le compte de l'AMF ne suffit plus à la tâche. En février 2018, il y avait plusieurs centaines d'entreprises qui étaient en attente de leur autorisation de renouvellement. Ces dernières peuvent, toutefois, se voir attribuer de lucratifs contrats publics, sans que l'UPAC ait vérifié s’il y a eu des changements au sein de l'entreprise. Lévesque, K. (2018).

17 En date du 7 septembre 2016, plus de 350 entreprises se sont vues inscrites au RENA. Voir le site repéré à https://rena. tresor.gouv.qc.ca/rena/rechercher.aspx?type $=$ lettre\&lettre $=a-z$ De nombreuses entreprises n'ont pas cru pouvoir obtenir cette autorisation.

18 Projet de loi $\mathrm{n}^{\circ}$ 98, Loi modifiant diverses lois concernant principalement l'admission aux professions et la gouvernance du système professionnel, Éditeur officiel du Québec, 2017. Repéré à http:/ /www2.publicationsduquebec.gouv.qc.ca/ dynamicSearch $/$ telecharge.php?type $=5 \&$ file $=2017$ C11F.PDF

19 On entend par soft law, des normes alternatives ou émergentes élaborées de façon incrémentale par les acteurs privés afin de combler « les interstices laissés libres par les droits nationaux et le droit international dans la régulation des phénomènes transnationaux» (Benyekhlef, 2016, p. 13).

20 Nos recherches ne nous ont pas permis de trouver le nombre d'entreprises canadiennes ayant des activités à l'étranger qui ont adopté des codes de conduite, accompagnés de programmes spécifiques d'application et de formation, afin de combattre la corruption.

21 La plupart des codes exposent la politique de l'entreprise relativement aux cadeaux ou avantages de toutes sortes pouvant être donnés ou reçus dans le cadre des relations interpersonnelles qui se nouent avec les agents publics ou privés étrangers. Ils précisent aussi les règles à l'égard de contributions aux partis politiques et à la caisse électorale (comptes de campagne) des candidats à des fonctions publiques.

22 On retrouve souvent dans les codes un article mentionnant que l'entreprise va se conformer à la législation contrant la corruption dans tous les territoires où elle exerce ses activités, notamment la Loi canadienne sur la corruption d'agents publics étrangers et ses amendements, la loi américaine contre la corruption dans le commerce international (U.S. Foreign Corrupt Practices Act) et la loi britannique relative à la répression et la prévention de la corruption (UK Bribery Act). 
23 Créée à Paris en 1919, l'ICC compte parmi ses membres des milliers d'entreprises et d'organisations présentes dans 130 pays.

24 Partie II des règles (Corporate Policies to Support Compliance with the Anti-Corruption Rules), en particulier les articles 3 à 5.

25 En particulier : ICC Guidelines on Whistleblowing; ICC Guidelines on Agents, Intermediaries and Other Third Parties, 2010; ICC Guidelines on Gifts and Hospitality, 2014.

26 Pour la conception de leur code de conduite, les entreprises peuvent aussi puiser à l'OCDE, spécialement à l'annexe II de la recommandation du Conseil du 26 novembre 2009, et intitulée Guide de bonnes pratiques pour les contrôles internes, la déontologie et la conformité (OCDE, 2010).

27 Selon certains observateurs, seulement $10 \%$ des entreprises multinationales ont adhéré au Pacte mondial et à ses principes.

28 Pour une liste des entreprises qui ont fait l'objet d'un retrait du Pacte mondial des Nations Unies, on consultera : https:// www.unglobalcompact.org/participation/report/cop/create-and-submit/expelled?page=2\&per_page=250\#paged_results

29 https://www.transparency.org/whatwedo/tools/integrity_pacts

\section{RÉFÉRENCES}

Agence française anticorruption (2017). Rapport annuel d'activité 2017. Repéré à https://www.economie.gouv.fr/files/files/directions services/afa/Rapport annuel_ 22 mai_2018.pdf

Agence France-Presse (2018, 22 avril). Le FMI va évaluer de manière « plus systémique » la corruption. La Presse.ca. Repéré à http://www.lapresse.ca/affaires/economie/international/201804/22/01-5162015-le-fmi-va-evaluer-de-maniere-plussystematique-la-corruption.php

Akbar, Y. H. et Vujić, V. (2014). Explaining corruption: The role of national culture and its implication for international management. Cross Cultural Management, 21(2), 191-218.

Ampratwum, E. F. (2008). The fight against corruption and its implications for development in developing and transition economies. Journal of Money Laundering Control, 11(1), 76-87.

Anand, V., Ashforth, B. E. et Joshi, M. (2005). Business as usual: The acceptance and perpetuation of corruption in organizations. Academy of Management Executive, 19(4), 9-23.

Atcha, E. (2018, 27 janvier). La corruption fait perdre à l'Afrique le quart de son PIB. La Tribune Afrique. Repéré à https://afrique.latribune.fr/politique/gouvernance/2018-01-27/en-afrique-la-corruption-detruit-le-quart-du-pib766302.html

Barkemeyer, R., Preuss, L. et Lee, L. (2015). Corporate reporting on corruption: An international comparison. Accounting Forum, 39(4), 349-365.

Becklumb, P. (2007). Projet de loi C-48 : Loi modifiant le Code criminel en vue de la mise en cuuve de la convention des Nations Unies contre la corruption. Repéré à https://lop.parl.ca/Content/LOP/LegislativeSummaries/39/1/c48-f.pdf

Benyekhlef, K. (2016), Vers un droit global? Montréal, Québec : Éditions Thémis.

Blyschak, P. (2014). Corporate liability for foreign corrupt practices under Canadian law. Revue de Droit de McGill, 59(3), $655-705$.

Bonell, M.-J. et Meyer, O. (2015). The impact of corruption on international commercial contracts - General report. Dans M.-J. Bonell et O. Meyer. (dir.). The impact of corruption on international commercial contracts, (Ius Comparatum - Global Studies in Comparative Law, vol. 11, p. 2). Cham, Suisse : Springer.

Boyer, R. (2015). Économie politique des capitalismes. Théorie de la régulation et des crises. Paris, France : La Découverte.

Cavalerie, Ph. (1997). La convention OCDE du 17 décembre 1997 sur la lutte contre la corruption d'agents publics étrangers dans les transactions commerciales internationales. Annuaire français de droit international, 43, 609-632.

CleanGovBiz (2013). The rationale for fighting corruption. Background brief. Repéré à https://www.oecd.org/cleangovbiz/49693613.pdf

Commission d'ICC sur la responsabilité sociale de l'entreprise et la lutte contre la corruption. (2011). Règles d'ICC pour combattre la corruption. Repéré à https://cdn.iccwbo.org/content/uploads/sites/3/2011/12/RC3A8gles-dE28099ICC-pourcombattre-la-corruption.pdf 
Conférence des Nations Unies sur le commerce et le développement. (2017). Rapport sur l'investissement dans le monde 2017. L'investissement et l'économie numérique. Repéré à http://unctad.org/fr/PublicationsLibrary/wir2017 overview fr.pdf

Côté-Harper, G., Rainville, P. et Turgeon, J. (1998). Traité de droit pénal canadien (4e éd.). Cowansville, Québec : Éditions Yvon Blais.

Dattu, R., Ritchie, L. et Pavic, S. (2018, juillet). Deferred prosecution agreements to be introduced in Canada. Financier Worldwide Magazine. Repéré à https://www.financierworldwide.com/deferred-prosecution-agreements-to-be-introducedin-canada\#.W0TInWa2 y

Desbarats, I. (2003). Codes de conduite et chartes éthiques des entreprises privées, regard sur une pratique en expansion. La Semaine Juridique - Édition Générale, (9), 337-343.

Decaux, E. et Frouville, de O. (2012). Droit international public. (8e éd.). Paris, France : Dalloz.

D’Hollander, J. (1999). Ethics in business : The new OECD convention on bribery. Revue Juridique Thémis, 33, 147-1665.

Dion, M. (2013). Uncertainties and presumptions about corruption. Social Responsibility Journal, 9(3), 412-426.

Eljarrat, S. et Bodrug, J. (2018). La législation anticorruption au Canada. La corruption à l'étranger. Repéré à https://www.dwpv.com/fr/Insights/Publications/2018/Anti-Corruption-Legislation-in-Canada

Ford, R. C. et Richardson, W. D. (1994). Ethical decision making: A review of the empirical literature. Journal of Business Ethics, 13(3), 205-221.

Heimann, F., Földes, À. et Coles, S. (2015). Exporting corruption, progress report 2015: Assessing enforcement of the OECD convention on combatting foreign bribery. Repéré à https://www.transparency.org/whatwedo/publication/exporting corruption progress report 2015 assessing enforcement of the oecd

G20 (2016). G20 anti-corruption action plan 2017-2018. Repéré à http://www.mofa.go.jp/files/000185882.pdf

G20 Anti-Corruption Working Group. (2017). Anti-corruption working group interim report 2017. Repéré à https://www.g20.org/sites/default/files/media/g20 acwg interim report.pdf

Garapon, A. (2014). La peur de l'impuissance démocratique. Esprit, 2(février), 19-30.

Girard, D. (2007). Culture organisationnelle, contexte d'affaires et prise de décision éthique. Gestion, 32(1), 101-111. doi.org/10.3917/riges.321.0101

Global Reporting Initiative (2013). G4. Lignes directrices pour le reporting développement durable. Principes de reporting et éléments d'information. Repéré à http://www.ecpar.org/files/documents/french-g4-part-one.pdf

Gosselin, I. et Pellerin, A. (2013). Les contrats publics au Québec : une question d'intégrité. La nouvelle Loi sur l’intégrité en matière de contrats publics. CCCL Journal, 15-28.

Gouvernement du Canada. (2017). La lutte du Canada contre la corruption transnationale, Dix-buitième rapport annuel au Parlement. Repéré à http://www.international.gc.ca/trade-agreements-accords-commerciaux/topics-frdomaines/other-autre/ corr-18.aspx?lang=fra

Groupe consultatif à haut niveau. (2017). Rapport sur la lutte contre la corruption et la promotion de l'intégrité présenté au Secrétaire général de l'OCDE. Repéré à http://www.oecd.org/fr/corruption/anti-corruption/HLAG-Corruption-Intégrité-Rapport-Mars2017.pdf

Harrison, A. (2014). Business environment in a global context (2e éd.). Oxford, Royaume-Uni: Oxford University Press.

Heimann, F. et Vincke, F. (2008). Fighting corruption - International corporate integrity handbook. (3e éd.). Paris, France: International Chamber of Commerce.

Heywood, P. M. et Rose, J. (2014). Close but no cigar: the measurement of corruption. Journal of Public Policy, 34(3), 507-529.

Husted, B. W. (1999). Wealth, culture, and corruption. Journal of International Business Studies, 30(2), 339-359.

International Monetary Fund (2016). Corruption: Costs and mitigating strategies. Repéré à https://www.imf.org/en/ Publications/Staff-Discussion-Notes/Issues/2016/12/31/Corruption-Costs-and-Mitigating-Strategies-43888

International Chamber of Commerce (2012). ICC anti-corruption clause. Repéré à https://iccwbo.org/publication/ icc-anti-corruption-clause 
International Organization for Standardization (2016). ISO 37001: 2016 Anti-bribery management systems - Requirements with guidance for use.

Jose, A. et Thibodeaux, M. S. (1999). Institutionalization of ethics: The perspective of managers. Journal of Business Ethics, 22(2), $133-143$.

Johnston, M. (1996). The search for definition: The vitality of politics and the issues of corruption. International Social Science Journal, 48(149), 321-335.

Katz, K. (2011). Here comes the bribe: Canada's efforts to combat corruption in international business. Advocate (V ancouver), 69, 501.

Keith, N. (2017). Canadian anti-corruption law and compliance (2e éd.). Montréal, Québec, Toronto, Ontario : LexisNexis Canada.

Köbis N. C., van Prooijen, J.-W., Righetti, F. et Van Lange, P. A. M. (2015). “Who doesn’t?”- The impact of descriptive norms on corruption. PLOS ONE, 10(6). doi.org/10.1371/journal.pone.0131830

Lane, H. W. et Simpson, D. G. (1984). Bribery in international business: Whose problem is it? Journal of Business Ethics, 3(1), 35-42.

Lévesque, K. (2018, 21 février). Des millions à des entreprises en attente du feu vert de l'UPAC. La Presse.ca. Repéré à http://www.lapresse.ca/actualites/national/201802/20/01-5154656-des-millions-a-des-entreprises-en-attente-du-feu-vertde-lupac.php

Litzky, B. E., Eddleston, K. A. et Kidder, D. L. (2006). The good, the bad, and the misguided: How managers inadvertently encourage deviant behaviors. The Academy of Management Perspectives, 20(1), 91-103.

Loe, T. W., Ferrell, L. et Mansfield, P. (2000). A review of empirical studies assessing ethical decision making in business. Journal of Business Ethics, 25(3), 185-204.

Martini, M. et Murphy, M. (2018, avril). G20 leaders or laggards? Reviewing G20 promises on ending anonymous companies. Repéré à https://www.transparency.org/whatwedo/publication/g20 leaders or laggards

Mauro, P. (1997). Why worry about corruption? Repéré à https://projects.iq.harvard.edu/gov2126/files/mauro 1997.pdf

Ménissier, T. (2007). La corruption, un concept philosophique et politique chez les Anciens et les Modernes. Anabases, 6, 11-16.

Montesquieu (1772). De l'esprit des lois : Tome premier. Repéré à http://hdl.handle.net/2027/ucm.5326517021

Morin, F. (2015). L’hydre mondiale. L’oligopole bancaire. Montréal, Québec : Lux éditeur.

O'Fallon, M. J. et Butterfield, K. D. (2005). A review of the empirical ethical decision-making literature: 1996-2003. Journal of Business Ethics, 59(4), 375-413.

OCDE (2001). La lutte contre les pots-de-vin et la corruption. L'Observateur de l'OCDE, (janvier), 7.

OCDE (2006). Bilan à mi-parcours des rapports au titre de la phase 2. Mise en cuvre de la Convention sur la lutte contre la corruption d'agents publics étrangers dans les transactions commerciales internationales et de la recommandation de 1997 sur la lutte contre la corruption dans les transactions commerciales internationales. Groupe de travail sur la corruption Repéré à http://www.oecd.org/fr/daf/ anti-corruption/conventioncontrelacorruption/36872265.pdf

OCDE (2010). Guide de bonnes pratiques pour les contrôles internes, la déontologie et la conformité. Repéré à https://www.oecd.org/fr/daf/anti-corruption/conventioncontrelacorruption/44884414.pdf

OCDE (2011a). Convention sur la lutte contre la corruption d'agents publics étrangers dans les transactions commerciales internationales et documents connexes. Repéré à http://www.oecd.org/fr/daf/anti-corruption/ConvCombatBribery FR.pdf

OCDE (2011b). Rapport de phase 3 sur la mise en ceuvre par le Canada de la Convention de l'OCDE sur la lutte contre la corruption. Repéré à http://www.oecd.org/fr/daf/anti-corruption/conventioncontrelacorruption/CanadaPhase3FR.pdf

OCDE (2014). Rapport de l'OCDE sur la corruption transnationale : une analyse de l'infraction de corruption d'agents publics étrangers. doi.org/10.1787/9789264226623-fr

Office des Nations Unies contre la drogue et le crime. (2004). Convention des Nations Unies contre la corruption. Repéré à https://www.unodc.org/documents/treaties/UNCAC/Publications/Convention/08-50027_F.pdf 
ONU (n/d). Journée internationale de la lutte contre la corruption. Repéré à http://www.un.org/fr/events/anticorruptionday/ $\underline{\text { convention.shtml }}$

ONU (2000). Global Compact - Le Pacte mondial. Repéré à https://www.diplomatie.gouv.fr/sites/odyssee-developpementdurable/files $/ 17 / 10$ principes pacte mondial.pdf

Perrin, B. (2010). Le rôle de l'éthique dans la prévention de la corruption. Le cas de la Suisse. Sécurité et stratégie 2010/HS1 (3), 87-94. doi:10.3917/sestr.hs1.0087

Perrot, E. (2016, janvier). Une dette « odieuse ». Corruption. [Billet de blogue]. Repéré à https://www.jesuites.ch/chroniques/le-coup-d-epingle-d-etienne-perrot-sj/43-archives/884-annee-2016

Quinones, E. (2000). Qu'est-ce que la corruption? L'Observateur, 220(avril), 21-22.

Quinones E. (2003). L'évolution du droit international en matière de corruption : la convention de l'OCDE. Annuaire français de droit international, 49, 563-574.

Rabl, T. (2011). The impact of situational influences on corruption in organizations. Journal of Business Ethics, 100(1), 85-101. Repéré à https://www.jstor.org/stable/41475829

Robinson, M. (dir.) (2004). Corruption and development: An introduction, London, Royaume-Uni: Routledge.

Rodriguez, P., Siegel, D. S., Hillman, A. et Eden, L. (2006). Three lenses on the multinational enterprise: Politics, corruption, and corporate social responsibility. J. Int. Bus. Stud., 37(6), 733-746.

Sancerre, O. (2016, 12 mai). Corruption : un mal qui dévore le PIB mondial. Le Journal de l'économie. Repéré à https://www.journaldeleconomie.fr/Corruption-un-mal-qui-devore-le-PIB-mondial a3515.html

Sanyal, R. (2005). Determinants of bribery in international business: The cultural and economic factors. Journal of Business Ethics, 59(1-2), 139-145.

Schein, E. H. (1985). Organizational culture and leadership: A dynamic view. San Francisco, CA: Jossey-Brass.

Transparency International. (2017, février). Corruption perceptions index 2017. Repéré à https://www.transparency.org/news/ feature/corruption perceptions index 2017

Tomasic, R. (2018). Global corporations, bribery and corrupt practices: Anti-bribery laws and the limits of state action, Law and Financial Markets Review, 12(1), 18-30. doi: 10.1080/17521440.2018.1435459

Treviño, L. K., Weaver, G. R., Gibson, D. G. et Toffler, B. L. (1999). Managing ethics and legal compliance: What works and what hurts. California Management Review, 41(2), 131-151.

Vincke, F. et Kassum, J. (2013). ICC ethics and compliance training bandbook - Anti-corruption guidance for practitioners by practitioners. ICC Publication.

Vitali, S., Glattfelder, J. B. et Battiston, S. (2011). The network of global corporate control. PLOS ONE, 6(10). doi.org/10.1371/journal.pone.0025995

Wei, S.-J. (2000). How taxing is corruption on international investors? Review of Economics and Statistics, 82(février), 1-11. 